\title{
A Constrained Least Squares Approach to Mobile Positioning: Algorithms and Optimality
}

\author{
K. W. Cheung, ${ }^{1}$ H. C. So, ${ }^{1}$ W.-K. Ma, ${ }^{2}$ and Y. T. Chan $^{3}$ \\ ${ }^{1}$ Department of Electronic Engineering, City University of Hong Kong, Tat Chee Avenue, Kowloon, Hong Kong \\ ${ }^{2}$ Department of Electrical Engineering, National Tsing Hua University, Hsinchu 30013, Taiwan \\ ${ }^{3}$ Department of Electrical \& Computer Engineering, Royal Military College of Canada, Kingston, ON, Canada K7K 7B4
}

Received 20 May 2005; Revised 25 November 2005; Accepted 8 December 2005

\begin{abstract}
The problem of locating a mobile terminal has received significant attention in the field of wireless communications. Time-ofarrival (TOA), received signal strength (RSS), time-difference-of-arrival (TDOA), and angle-of-arrival (AOA) are commonly used measurements for estimating the position of the mobile station. In this paper, we present a constrained weighted least squares (CWLS) mobile positioning approach that encompasses all the above described measurement cases. The advantages of CWLS include performance optimality and capability of extension to hybrid measurement cases (e.g., mobile positioning using TDOA and AOA measurements jointly). Assuming zero-mean uncorrelated measurement errors, we show by mean and variance analysis that all the developed CWLS location estimators achieve zero bias and the Cramér-Rao lower bound approximately when measurement error variances are small. The asymptotic optimum performance is also confirmed by simulation results.
\end{abstract}

Copyright $\odot 2006$ Hindawi Publishing Corporation. All rights reserved.

\section{INTRODUCTION}

Accurate positioning of a mobile station (MS) will be one of the essential features that assists third generation (3G) wireless systems in gaining a wide acceptance and triggering a large number of innovative applications. Although the main driver of location services is the requirement of locating Emergency 911 (E-911) callers within a specified accuracy in the United States [1], mobile position information will also be useful in monitoring of the mentally impaired (e.g., the elderly with Alzheimer's disease), young children and parolees, intelligent transport systems, location billing, interactive map consultation and location-dependent e-commerce [2-6]. Global positioning system (GPS) could be used to provide mobile location, however, it would be expensive to be adopted in the mobile phone network because additional hardware is required in the MS. Alternatively, utilizing the base stations (BSs) in the existing network for mobile location is preferable and is more cost effective for the consumer. The basic principle of this softwarebased solution is to use two or more BSs to intercept the MS signal, and common approaches [6-8] are based on time-of-arrival (TOA), received signal strength (RSS), time-difference-of-arrival (TDOA), and/or angle-of-arrival (AOA) measurements determined from the MS signal received at the BSs.
In the TOA method, the distance between the MS and BS is determined from the measured one-way propagation time of the signal traveling between them. For two-dimensional (2D) positioning, this provides a circle centered at the BS on which the MS must lie. By using at least three BSs to resolve ambiguities arising from multiple crossings of the lines of position, the MS location estimate is determined by the intersection of circles. The RSS approach employs the same trilateration concept where the propagation path losses from the MS to the BSs are measured to give their distances. In the TDOA method, the differences in arrival times of the MS signal at multiple pairs of BSs are measured. Each TDOA measurement defines a hyperbolic locus on which the MS must lie and the position estimate is given by the intersection of two or more hyperbolas. Finally, the AOA method necessitates the BSs to have multielement antenna arrays for measuring the arrival angles of the transmitted signal from the MS at the BSs. From each AOA estimate, a line of bearing (LOB) from the BS to the MS can be drawn and the position of the MS is calculated from the intersection of a minimum of two LOBs. In general, the MS position is not determined geometrically but is estimated from a set of nonlinear equations constructed from the TOA, RSS, TDOA, or AOA measurements, with knowledge of the BS geometry.

Basically, there are two approaches for solving the nonlinear equations. The first approach [9-12] is to solve them 
directly in a nonlinear least squares (NLS) or weighted least squares (WLS) framework. Although optimum estimation performance can be attained, it requires sufficiently precise initial estimates for global convergence because the corresponding cost functions are multimodal. The second approach [13-17] is to reorganize the nonlinear equations into a set of linear equations so that real-time implementation is allowed and global convergence is ensured. In this paper, the latter approach is adopted, and we will focus on a unified development of accurate location algorithms, given the TOA, RSS, TDOA, and/or AOA measurements.

For TDOA-based location systems, it is well known that for sufficiently small noise conditions, the corresponding nonlinear equations can be reorganized into a set of linear equations by introducing an intermediate variable, which is a function of the source position, and this technique is commonly called spherical interpolation (SI) [13]. However, the SI estimator solves the linear equations via standard least squares (LS) without using the known relation between the intermediate variable and the position coordinate. To improve the location accuracy of the SI approach, Chan and Ho have proposed [14] to use a two-stage WLS to solve for the source position by exploiting this relation implicitly via a relaxation procedure, while [15] incorporates the relation explicitly by minimizing a constrained LS function based on the technique of Lagrange multipliers. According to [15], these two modified algorithms are referred to as the quadratic correction least squares (QCLS) and linear correction least squares (LCLS), respectively. Recently, we have improved [18] the performance of the LCLS estimator by introducing a weighting matrix in the optimization, which can be regarded as a hybrid version of the QCLS and LCLS algorithms. The idea of this constrained weighted least squares (CWLS) technique has also been extended to the RSS [19] and TOA [20] measurements. Using a different way of converting nonlinear equations to linear equations without introducing dummy variables, Pages-Zamora et al. [16] have developed a simple LS AOA-based location algorithm. In this work, our contributions include (i) development of a unified approach for mobile location which allows utilizing different combinations of TOA, RSS, TDOA, and AOA measurements via generalizing [18-20] and improving [16] with the use of WLS; and (ii) derivation of bias and variance expressions for all the proposed algorithms. In particular, we prove that the performance of all the proposed estimation methods can achieve zero bias and the Cramér-Rao lower bound (CRLB) [21] approximately when the measurement errors are uncorrelated and small in magnitude.

The rest of this paper is organized as follows. In Section 2, we formulate the models for the TOA, TDOA, RSS, and AOA measurements and state our assumptions. In Section 3, three CWLS location algorithms using TDOA, RSS, and TOA measurements, respectively, are first reviewed, and a WLS AOA-based location algorithm is then devised via modifying [16]. Mobile location using various combinations of TOA, TDOA, RSS, and AOA measurements is also examined. In particular, a TDOA-AOA hybrid algorithm is presented in detail. The performance of all the developed algorithms
TABLE 1: List of abbreviations and symbols.

\begin{tabular}{ll}
\hline AOA & Angle-of-arrival \\
CWLS & Constrained weighted least squares \\
CRLB & Cramér-Rao lower bound \\
NLS & Nonlinear least squares \\
RSS & Received signal strength \\
TOA & Time-of-arrival \\
TDOA & Time-difference-of-arrival \\
$\mathbf{A}^{T}$ & Transpose of matrix A \\
$\mathbf{A}^{-1}$ & Inverse of matrix $\mathbf{A}$ \\
$\mathbf{A}^{o}$ & Optimum matrix of $\mathbf{A}$ \\
$\sigma^{2}$ & Noise variance \\
$\mathbf{C}_{\mathbf{n}}$ & Noise covariance matrix \\
$\mathbf{I}(\mathbf{x})$ & Fisher information matrix for parameter vector $\mathbf{x}$ \\
$\tilde{\mathbf{x}}$ & Optimization variable vector for $\mathbf{x}$ \\
$\widehat{\mathbf{x}}$ & Estimate of $\mathbf{x}$ \\
diag $(\mathbf{x})$ & Diagonal matrix formed from vector $\mathbf{x}$ \\
$\mathbf{I}_{M}$ & $M \times M$ identity matrix \\
$\mathbf{1}_{M}$ & $M \times 1$ column vector with all ones \\
$\mathbf{0}_{M}$ & $M \times 1$ column vector with all zeros \\
$\mathbf{O}_{M \times N}$ & $M \times N$ matrix with all zeros \\
$\odot$ & Element-by-element multiplication \\
\hline
\end{tabular}

is studied in Section 4. Simulation results are presented in Section 5 to evaluate the location estimation performance of the proposed estimators and verify our theoretical findings. Finally, conclusions are drawn in Section 6. A list of abbreviations and symbols that are used in the paper is given in Table 1.

\section{MEASUREMENT MODELS}

In this section, the models and assumptions for the TOA, TDOA, RSS, and AOA measurements are described. Let $\mathbf{x}=$ $[x, y]^{T}$ be the MS position to be determined and let the known coordinates of the $i$ th BS be $\mathbf{x}_{i}=\left[x_{i}, y_{i}\right]^{T}, i=1,2$, $\ldots, M$, where the superscript $T$ denotes the transpose operation and $M$ is the total number of receiving BSs. The distance between the MS and the $i$ th BS, denoted by $d_{i}$, is given by

$$
d_{i}=\sqrt{\left(x-x_{i}\right)^{2}+\left(y-y_{i}\right)^{2}}, i=1,2, \ldots, M
$$

\subsection{TOA measurement}

The TOA is the one-way propagation time taken for the signal to travel from the MS to a BS. In the absence of disturbance, the TOA measured at the $i$ th BS, denoted by $t_{i}$, is

$$
t_{i}=\frac{d_{i}}{c}, \quad i=1,2, \ldots, M
$$


where $c$ is the speed of light. The range measurement based on $t_{i}$ in the presence of disturbance, denoted by $r_{\mathrm{TOA}, i}$, is modeled as

$$
\begin{aligned}
r_{\mathrm{TOA}, i} & =d_{i}+n_{\mathrm{TOA}, i} \\
& =\sqrt{\left(x-x_{i}\right)^{2}+\left(y-y_{i}\right)^{2}}+n_{\mathrm{TOA}, i}, \quad i=1,2, \ldots, M,
\end{aligned}
$$

where $n_{\mathrm{TOA}, i}$ is the range error in $r_{\mathrm{TOA}, i}$. Equation (3) can also be expressed in vector form as

$$
\mathbf{r}_{\mathrm{TOA}}=\mathbf{f}_{\mathrm{TOA}}(\mathbf{x})+\mathbf{n}_{\mathrm{TOA}},
$$

where

$$
\begin{aligned}
\mathbf{r}_{\mathrm{TOA}}= & {\left[r_{\mathrm{TOA}, 1} r_{\mathrm{TOA}, 2} \cdots r_{\mathrm{TOA}, M}\right]^{T}, } \\
\mathbf{n}_{\mathrm{TOA}}= & {\left[n_{\mathrm{TOA}, 1} n_{\mathrm{TOA}, 2} \cdots n_{\mathrm{TOA}, M}\right]^{T}, } \\
\mathbf{f}_{\mathrm{TOA}}(\mathbf{x})= & {\left[\begin{array}{c}
\sqrt{\left(x-x_{1}\right)^{2}+\left(y-y_{1}\right)^{2}} \\
\sqrt{\left(x-x_{2}\right)^{2}+\left(y-y_{2}\right)^{2}} \\
\vdots \\
\sqrt{\left(x-x_{M}\right)^{2}+\left(y-y_{M}\right)^{2}}
\end{array}\right] . }
\end{aligned}
$$

\subsection{TDOA measurement}

The TDOA is the difference in TOAs of the MS signal at a pair of BSs. Assigning the first BS as the reference, it can be easily deduced that the range measurements based on the TDOAs are of the form

$$
\begin{aligned}
r_{\mathrm{TDOA}, i}= & \left(d_{i}-d_{1}\right)+n_{\mathrm{TDOA}, i} \\
= & \sqrt{\left(x-x_{i}\right)^{2}+\left(y-y_{i}\right)^{2}}-\sqrt{\left(x-x_{1}\right)^{2}+\left(y-y_{1}\right)^{2}} \\
& +n_{\mathrm{TDOA}, i}, \quad i=2,3, \ldots, M,
\end{aligned}
$$

where $n_{\mathrm{TDOA}, i}$ is the range error in $r_{\mathrm{TDOA}, i}$. Notice that if the TDOA measurements are directly obtained from the TOA data, then $n_{\mathrm{TDOA}, i}=n_{\mathrm{TOA}, i}-n_{\mathrm{TOA}, 1}, i=2,3, \ldots, M$. In vector form, (6) becomes

$$
\mathbf{r}_{\mathrm{TDOA}}=\mathbf{f}_{\mathrm{TDOA}}(\mathbf{x})+\mathbf{n}_{\mathrm{TDOA}},
$$

where

$$
\begin{aligned}
\mathbf{r}_{\mathrm{TDOA}} & =\left[r_{\mathrm{TDOA}, 2} r_{\mathrm{TDOA}, 3} \cdots r_{\mathrm{TDOA}, M}\right]^{T}, \\
\mathbf{n}_{\mathrm{TDOA}} & =\left[n_{\mathrm{TDOA}, 2} n_{\mathrm{TDOA}, 3} \cdots n_{\mathrm{TDOA}, M}\right]^{T}, \\
\mathbf{f}_{\mathrm{TDOA}}(\mathbf{x}) & =\left[\begin{array}{c}
\sqrt{\left(x-x_{2}\right)^{2}+\left(y-y_{2}\right)^{2}}-\sqrt{\left(x-x_{1}\right)^{2}+\left(y-y_{1}\right)^{2}} \\
\sqrt{\left(x-x_{3}\right)^{2}+\left(y-y_{3}\right)^{2}}-\sqrt{\left(x-x_{1}\right)^{2}+\left(y-y_{1}\right)^{2}} \\
\vdots \\
\sqrt{\left(x-x_{M}\right)^{2}+\left(y-y_{M}\right)^{2}}-\sqrt{\left(x-x_{1}\right)^{2}+\left(y-y_{1}\right)^{2}}
\end{array}\right] .
\end{aligned}
$$

\subsection{RSS measurement}

Without measurement error, the RSS or received power at the $i$ th BS, denoted by $P_{i}^{r}$, can be modeled as [22]

$$
P_{i}^{r}=K_{i} \frac{P_{i}^{t}}{d_{i}^{a}}, \quad i=1,2, \ldots, M,
$$

where $P_{i}^{t}$ is the transmitted power, $K_{i}$ accounts for all other factors which affect the received power, including the antenna height and antenna gain, and $a$ is the propagation constant. Note that the propagation parameter $a$ can be obtained via finding the path loss slope by measurement [22]. In free space, $a$ is equal to 2, but in some urban and suburban areas, $a$ can vary from 3 to 6 . From (9), the range measurements based on the RSS data with the use of the known $\left\{P_{i}^{t}\right\}$ and $\left\{K_{i}\right\}$, denoted by $\left\{r_{\mathrm{RSS}, i}\right\}$, are determined as

$$
\begin{aligned}
r_{\mathrm{RSS}, i} & =K_{i} \frac{P_{i}^{t}}{P_{i}^{r}}+n_{\mathrm{RSS}, i} \\
& =\left[\left(x-x_{i}\right)^{2}+\left(y-y_{i}\right)^{2}\right]^{a / 2}+n_{\mathrm{RSS}, i}, \quad i=1,2, \ldots, M,
\end{aligned}
$$

where $n_{\mathrm{RSS}, i}$ is the range error in $r_{\mathrm{RSS}, i}$. It is noteworthy that if $a=1$, then (10) will be of the same form as (3). Equation (10) can also be expressed in vector form as

$$
\mathbf{r}_{\mathrm{RSS}}=\mathbf{f}_{\mathrm{RSS}}(\mathbf{x})+\mathbf{n}_{\mathrm{RSS}},
$$

where

$$
\begin{aligned}
& \mathbf{r}_{\mathrm{RSS}}=\left[r_{\mathrm{RSS}, 1} r_{\mathrm{RSS}, 2} \cdots r_{\mathrm{RSS}, M}\right]^{T}, \\
& \mathbf{n}_{\mathrm{RSS}}=\left[n_{\mathrm{RSS}, 1} n_{\mathrm{RSS}, 2} \cdots n_{\mathrm{RSS}, M}\right]^{T},
\end{aligned}
$$$$
\mathbf{f}_{\mathrm{RSS}}(\mathbf{x})=\left[\begin{array}{c}
{\left[\left(x-x_{1}\right)^{2}+\left(y-y_{1}\right)^{2}\right]^{a / 2}} \\
{\left[\left(x-x_{2}\right)^{2}+\left(y-y_{2}\right)^{2}\right]^{a / 2}} \\
\vdots \\
{\left[\left(x-x_{M}\right)^{2}+\left(y-y_{M}\right)^{2}\right]^{a / 2}}
\end{array}\right] .
$$ 


\subsection{AOA measurement}

The AOA of the transmitted signal from the MS at the ith BS, denoted by $\phi_{i}$, is related to $\mathbf{x}$ and $\mathbf{x}_{i}$ by

$$
\tan \left(\phi_{i}\right)=\frac{y-y_{i}}{x-x_{i}}, \quad i=1,2, \ldots, M .
$$

Geometrically, $\phi_{i}$ is the angle between the LOB from the $i$ th $\mathrm{BS}$ to the MS and the $x$-axis. The AOA measurements in the presence of angle errors, denoted by $\left\{r_{\mathrm{AOA}, i}\right\}$, are modeled as

$$
r_{\mathrm{AOA}, i}=\phi_{i}+n_{\mathrm{AOA}, i}=\tan ^{-1}\left(\frac{y-y_{i}}{x-x_{i}}\right)+n_{\mathrm{AOA}, i}, \quad i=1,2, \ldots, M,
$$

where $n_{\mathrm{AOA}, i}$ is the noise in $r_{\mathrm{AOA}, i}$. Equation (14) can also be expressed in vector form as

$$
\mathbf{r}_{\mathrm{AOA}}=\mathbf{f}_{\mathrm{AOA}}(\mathbf{x})+\mathbf{n}_{\mathrm{AOA}},
$$

where

$$
\begin{aligned}
\mathbf{r}_{\mathrm{AOA}}= & {\left[r_{\mathrm{AOA}, 1} r_{\mathrm{AOA}, 2} \cdots r_{\mathrm{AOA}, M}\right]^{T}, } \\
\mathbf{n}_{\mathrm{AOA}}= & {\left[n_{\mathrm{AOA}, 1} n_{\mathrm{AOA}, 2} \cdots n_{\mathrm{AOA}, M}\right]^{T}, } \\
\mathbf{f}_{\mathrm{AOA}}(\mathbf{x})= & {\left[\begin{array}{c}
\tan ^{-1}\left(\frac{y-y_{1}}{x-x_{1}}\right) \\
\tan ^{-1}\left(\frac{y-y_{2}}{x-x_{2}}\right) \\
\vdots \\
\tan ^{-1}\left(\frac{y-y_{M}}{x-x_{M}}\right)
\end{array}\right] . }
\end{aligned}
$$

To facilitate the development and analysis of the proposed location algorithms, we make the following assumptions for the TOA, TDOA, RSS, and AOA measurements.

(A1) All measurement errors, namely, $\left\{n_{\mathrm{TOA}, i}\right\},\left\{n_{\mathrm{TDOA}, i}\right\}$, $\left\{n_{\mathrm{RSS}, i}\right\}$, and $\left\{n_{\mathrm{AOA}, i}\right\}$ are sufficiently small and are modeled as zero-mean Gaussian random variables with known covariance matrices, denoted by $\mathbf{C}_{\mathbf{n}, \mathrm{TOA}}$, $\mathbf{C}_{\mathbf{n}, \mathrm{TDOA}}, \mathbf{C}_{\mathbf{n}, \mathrm{RSS}}$, and $\mathbf{C}_{\mathbf{n}, \mathrm{AOA}}$, respectively. The zeromean error assumption implies that multipath and non-line-of-sight (NLOS) errors have been mitigated, which can be done by considering the techniques in [23-27]. Nevertheless, the effect of NLOS propagation will be studied in Section 5 for the TOA measurements.

(A2) For RSS-based location, the propagation parameter $a$ is known and has a constant value for all RSS measurements.

(A3) The numbers of BSs for location using the TOA, TDOA, RSS, and AOA measurements are at least 3, 4, 3 , and 2 , respectively.

\section{ALGORITHM DEVELOPMENT}

This section describes our development of the CWLS/WLS mobile positioning approach for the cases of TDOA, RSS, TOA, and AOA measurements. We also discuss how the proposed methods can be extended to hybrid measurement cases, such as the TDOA-AOA.

\subsection{TDOA [18]}

Without disturbance, (6) becomes

$$
\begin{aligned}
r_{\mathrm{TDOA}, i} & =\sqrt{\left(x-x_{i}\right)^{2}+\left(y-y_{i}\right)^{2}}-\sqrt{\left(x-x_{1}\right)^{2}+\left(y-y_{1}\right)^{2}} \\
& \Longrightarrow r_{\mathrm{TDOA}, i}+\sqrt{\left(x-x_{1}\right)^{2}+\left(y-y_{1}\right)^{2}} \\
& =\sqrt{\left(x-x_{i}\right)^{2}+\left(y-y_{i}\right)^{2}}, \quad i=2,3, \ldots, M .
\end{aligned}
$$

Squaring both sides of (17) and introducing an intermediate variable, $R_{1}$, which has the form

$$
R_{1}=d_{1}=\sqrt{\left(x-x_{1}\right)^{2}+\left(y-y_{1}\right)^{2}}
$$

we obtain the following set of linear equations [13]

$$
\begin{aligned}
& \left(x-x_{1}\right)\left(x_{i}-x_{1}\right)+\left(y-y_{1}\right)\left(y_{i}-y_{1}\right)+r_{\mathrm{TDOA}, i} R_{1} \\
& =\frac{1}{2}\left[\left(x_{i}-x_{1}\right)^{2}+\left(y_{i}-y_{1}\right)^{2}-r_{\mathrm{TDOA}, i}^{2}\right], \quad i=2,3, \ldots, M .
\end{aligned}
$$

Writing (19) in matrix form gives

$$
\mathbf{G} \vartheta=\mathbf{h},
$$

where

$$
\begin{aligned}
\mathbf{G} & =\left[\begin{array}{ccc}
x_{2}-x_{1} & y_{2}-y_{1} & r_{\mathrm{TDOA}, 2} \\
\vdots & \vdots & \vdots \\
x_{M}-x_{1} & y_{M}-y_{1} & r_{\mathrm{TDOA}, M}
\end{array}\right], \\
\mathbf{h} & =\frac{1}{2}\left[\begin{array}{c}
\left(x_{2}-x_{1}\right)^{2}+\left(y_{2}-y_{1}\right)^{2}-r_{\mathrm{TDOA}, 2}^{2} \\
\vdots \\
\left(x_{M}-x_{1}\right)^{2}+\left(y_{M}-y_{1}\right)^{2}-r_{\mathrm{TDOA}, M}^{2}
\end{array}\right],
\end{aligned}
$$

and the parameter vector $\boldsymbol{\vartheta}=\left[x-x_{1}, y-y_{1}, R_{1}\right]^{T}$ consists of the MS location as well as $R_{1}$. 
In the presence of measurement errors, the SI technique determines the MS position by simply solving (20) via standard LS, and the location estimate is found from [13]

$$
\begin{aligned}
\widehat{\boldsymbol{\vartheta}} & =\arg \min _{\breve{\mathfrak{\vartheta}}}(\mathbf{G} \breve{\mathfrak{\vartheta}}-\mathbf{h})^{T}(\mathbf{G} \breve{\vartheta}-\mathbf{h}) \\
& =\left(\mathbf{G}^{T} \mathbf{G}\right)^{-1} \mathbf{G}^{T} \mathbf{h},
\end{aligned}
$$

where $\breve{\mathfrak{\vartheta}}=\left[\breve{x}-x_{1}, \breve{y}-y_{1}, \breve{R_{1}}\right]^{T}$ is an optimization variable vector and ${ }^{-1}$ represents the matrix inverse, without utilizing the known relationship between $\breve{x}, \breve{y}$, and $\breve{R_{1}}$.

An improvement to the SI estimator is the LCLS method [15], which solves the LS cost function in (22) subject to the constraint of $\left(\breve{x}-x_{1}\right)^{2}+\left(\breve{y}-y_{1}\right)^{2}=\breve{R}_{1}^{2}$, or equivalently,

$$
\breve{\mathfrak{\vartheta}}^{T} \boldsymbol{\Sigma} \breve{\mathfrak{\vartheta}}=0
$$

where $\Sigma=\operatorname{diag}(1,1,-1)$.

On the other hand, Chan and Ho [14] have improved the SI estimator through two stages. In the first stage of the QCLS estimator, a coarse estimate is computed by minimizing a WLS function

$$
(\mathbf{G} \breve{\vartheta}-\mathbf{h})^{T} \mathbf{Y}^{-1}(\mathbf{G} \breve{\vartheta}-\mathbf{h})
$$

where $\Upsilon$ is a symmetric weighting matrix, which is a function of the estimate of $R_{1}$, denoted by $\hat{R}_{1}$. A better estimate of $\boldsymbol{\vartheta}$ is then obtained in the second stage via minimizing $\left(\breve{x}-x_{1}\right)^{2}+$ $\left(\breve{y}-y_{1}\right)^{2}-\breve{R}_{1}^{2}$ according to another WLS procedure. Since $\widehat{R}_{1}$ is not available at the beginning, normally a few iterations between the two stages are required to attain the best solution [15].

The idea of our CWLS estimator is to combine the key principles in the CWLS and LCLS methods, that is, the MS position estimate is determined by minimizing (24) subject to (23). For sufficiently small measurement errors, the inverse of the optimum weighting matrix $\mathbf{\Upsilon}^{-1}$ for the CWLS algorithm is found using the best linear unbiased estimator (BLUE) [21] as in [14]:

$$
\mathbf{Y}^{o}=\mathbf{s}_{1} \mathbf{s}_{1}^{T} \odot \mathbf{C}_{\mathbf{n}, \mathrm{TDOA}}
$$

where

$$
\mathbf{s}_{1}=\left[\begin{array}{c}
d_{2} \\
d_{3} \\
\vdots \\
d_{M}
\end{array}\right]=\left[\begin{array}{c}
d_{2}-d_{1}+R_{1} \\
d_{3}-d_{1}+R_{1} \\
\vdots \\
d_{M}-d_{1}+R_{1}
\end{array}\right]
$$

and $\odot$ denotes element-by-element multiplication. Since $\Upsilon$ contains the unknown $\left\{d_{i}\right\}$, we express $d_{i}=d_{i}-d_{1}+R_{1}$ and approximate $d_{i}-d_{1}$ by $r_{\mathrm{TDOA}, i}$ and thus an approximate version of $\mathbf{Y}^{o}$, namely, $\hat{\mathbf{s}}_{1} \hat{\mathbf{s}}_{1}^{T} \odot \mathbf{C}_{\mathbf{n}, \mathrm{TDOA}}$ with $\hat{\mathbf{s}}_{1}=\left[r_{\mathrm{TDOA}, 2}+\right.$ $\left.\hat{R}_{1} \cdots r_{\mathrm{TDOA}, M}+\hat{R}_{1}\right]^{T}$ is employed in practice.
Similar to [15], the CWLS problem is solved by using the technique of Lagrange multipliers and the Lagrangian to be minimized is

$$
\mathcal{L}_{\mathrm{TDOA}}(\breve{\boldsymbol{\vartheta}}, \eta)=(\mathbf{G} \breve{\boldsymbol{\vartheta}}-\mathbf{h})^{T} \mathbf{Y}^{-1}(\mathbf{G} \breve{\boldsymbol{\vartheta}}-\mathbf{h})+\eta \breve{\boldsymbol{\vartheta}}^{T} \Sigma \breve{\boldsymbol{\vartheta}}
$$

where $\eta$ is the Lagrange multiplier to be determined. The estimate of $\boldsymbol{\vartheta}$ is obtained by differentiating $\mathcal{L}_{\text {TDOA }}(\breve{\vartheta}, \eta)$ with respect to $\breve{\vartheta}$ and then equating the results to zero (see Appen$\operatorname{dix}$ A.1):

$$
\widehat{\boldsymbol{\vartheta}}=\left(\mathbf{G}^{T} \mathbf{Y}^{-1} \mathbf{G}+\eta \boldsymbol{\Sigma}\right)^{-1} \mathbf{G}^{T} \mathbf{Y}^{-1} \mathbf{h}
$$

where $\eta$ is found from the following 4-root equation:

$$
\sum_{i=1}^{3} \frac{\alpha_{i} \beta_{i}}{\left(\eta+\zeta_{i}\right)^{2}}=0
$$

and $\left\{\alpha_{i}\right\},\left\{\beta_{i}\right\}$, and $\left\{\zeta_{i}\right\}, i=1,2,3$, have been defined in Appendix A.1. The procedure for CWLS TDOA-based location is summarized as follows.

(i) Set $\mathbf{Y}=\mathbf{I}_{M-1}$, where $\mathbf{I}_{M-1}$ denotes the identity matrix of dimension $(M-1)$.

(ii) Find all roots of (29) by using a standard root finding algorithm. Then take only the real roots into consideration as the Lagrange multiplier is always real for a real optimization problem.

(iii) Put the real $\eta$ 's back to (28) and obtain subestimates of $\hat{\boldsymbol{\vartheta}}$. Then choose the solution $\hat{\boldsymbol{\vartheta}}$ from those subestimates which makes the expression $(\mathbf{G} \breve{\mathfrak{\vartheta}}-\mathbf{h})^{T} \mathbf{Y}^{-1}(\mathbf{G} \breve{\mathfrak{\vartheta}}-\mathbf{h})$ minimum.

(iv) Construct $\Upsilon$ according to (25) using the obtained $\hat{R}_{1}$ in step (iii). Then, repeat steps (ii) and (iii) until $\hat{\boldsymbol{\vartheta}}$ converges.

\subsection{RSS [19]}

Without measurement errors, (10) becomes

$$
r_{\mathrm{RSS}, i}=\left[\left(x-x_{i}\right)^{2}+\left(y-y_{i}\right)^{2}\right]^{a / 2}, \quad i=1,2, \ldots, M
$$

Extending the SI technique and taking power $2 / a$ on both sides of (30) yields

$$
\begin{aligned}
r_{\mathrm{RSS}, i}^{2 / a} & =R_{2}^{2}-2 x x_{i}-2 y y_{i}+\left(x_{i}^{2}+y_{i}^{2}\right) \\
& \Longrightarrow x_{i} x+y_{i} y-0.5 R_{2}^{2} \\
& =\frac{1}{2}\left(x_{i}^{2}+y_{i}^{2}-r_{i}^{2 / a}\right), \quad i=1,2, \ldots, M,
\end{aligned}
$$

where

$$
R_{2}=\sqrt{x^{2}+y^{2}}
$$


is the introduced intermediate variable in order to linearize (30) in terms of $x, y$, and $R_{2}^{2}$. Similar to the TDOA measurements, (31) can be expressed in matrix-vector form:

$$
\mathbf{A} \boldsymbol{\theta}=\mathbf{b}
$$

where

$$
\begin{gathered}
\mathbf{A}=\left[\begin{array}{ccc}
x_{1} & y_{1} & -0.5 \\
\vdots & \vdots & \vdots \\
x_{M} & y_{M} & -0.5
\end{array}\right], \quad \boldsymbol{\theta}=\left[\begin{array}{c}
x \\
y \\
R_{2}^{2}
\end{array}\right], \\
\mathbf{b}=\frac{1}{2}\left[\begin{array}{c}
x_{1}^{2}+y_{1}^{2}-r_{\mathrm{RSS}, 1}^{2 / a} \\
\vdots \\
x_{M}^{2}+y_{M}^{2}-r_{\mathrm{RSS}, M}^{2 / a}
\end{array}\right]
\end{gathered}
$$

The CWLS estimate of $\boldsymbol{\theta}$ is obtained by minimizing

$$
(\mathbf{A} \breve{\boldsymbol{\theta}}-\mathbf{b})^{T} \boldsymbol{\Psi}^{-1}(\mathbf{A} \breve{\boldsymbol{\theta}}-\mathbf{b}),
$$

where $\Psi^{-1}$ is the corresponding weighting matrix, subject to

$$
\mathbf{q}^{T} \breve{\boldsymbol{\theta}}+\breve{\boldsymbol{\theta}}^{T} \mathbf{P} \breve{\boldsymbol{\theta}}=0
$$

such that

$$
\breve{\boldsymbol{\theta}}=\left[\begin{array}{c}
\breve{x} \\
\breve{y} \\
\breve{R}^{2}
\end{array}\right], \quad \mathbf{P}=\left[\begin{array}{lll}
1 & 0 & 0 \\
0 & 1 & 0 \\
0 & 0 & 0
\end{array}\right], \quad \mathbf{q}=\left[\begin{array}{c}
0 \\
0 \\
-1
\end{array}\right]
$$

Here, (36) is a matrix characterization of the relation in (32).

The optimum value of $\Psi$ is also determined based on the BLUE as follows. For sufficiently small measurement errors, the value of $r_{\mathrm{RSS}, i}^{2 / a}$ can be approximated as

$$
\begin{aligned}
r_{\mathrm{RSS}, i}^{2 / a} & =\left(d_{i}^{a}+n_{\mathrm{RSS}, i}\right)^{2 / a} \\
& \approx d_{i}^{2}+\frac{2}{a}\left(d_{i}\right)^{2-a} n_{\mathrm{RSS}, i}, \quad i=1,2, \ldots, M
\end{aligned}
$$

As a result, the disturbance between the true and estimate of the squared distances is

$$
\varepsilon_{i}=r_{\mathrm{RSS}, i}^{2 / a}-d_{i}^{2} \approx \frac{2}{a}\left(d_{i}\right)^{2-a} n_{\mathrm{RSS}, i}, \quad i=1,2, \ldots, M
$$

In vector form, $\left\{\varepsilon_{i}\right\}$ is expressed as

$$
\boldsymbol{\varepsilon}=\left[\frac{2}{a}\left(d_{1}\right)^{2-a} n_{\mathrm{RSS}, 1}, \frac{2}{a}\left(d_{2}\right)^{2-a} n_{\mathrm{RSS}, 2}, \ldots, \frac{2}{a}\left(d_{M}\right)^{2-a} n_{\mathrm{RSS}, M}\right]^{T}
$$

The covariance matrix of the disturbance, which leads to the optimum weighting matrix, is thus of the form

$$
\Psi^{o}=E\left\{\boldsymbol{\varepsilon} \boldsymbol{\varepsilon}^{T}\right\}=\mathbf{s}_{2} \mathbf{s}_{2}^{T} \odot \mathbf{C}_{\mathbf{n}, \mathrm{RSS}}
$$

where

$$
\mathbf{s}_{2}=\left[\begin{array}{llll}
\frac{1}{a}\left(d_{1}\right)^{2-a} & \frac{1}{a}\left(d_{2}\right)^{2-a} & \cdots & \frac{1}{a}\left(d_{M}\right)^{2-a}
\end{array}\right]^{T} .
$$

Since $\boldsymbol{s}_{2}$ depends on the unknowns $\left\{d_{i}\right\}$, we use $\left\{r_{i}^{1 / a}\right\}$ instead of $\left\{d_{i}\right\}$ to form an estimate of $s_{2}$, denoted by $\hat{s}_{2}$, which is

$$
\widehat{\mathbf{s}}_{2}=\left[\begin{array}{llll}
\frac{1}{a} r_{\mathrm{RSS}, 1}^{2 / a-1} & \frac{1}{a} r_{\mathrm{RSS}, 2}^{2 / a-1} & \cdots & \frac{1}{a} r_{\mathrm{RSS}, M}^{2 / a-1}
\end{array}\right]^{T} .
$$

Minimizing (35) subject to (36) is equivalent to minimizing the Lagrangian

$$
\mathcal{L}_{\mathrm{RSS}}(\breve{\boldsymbol{\theta}}, \lambda)=(\mathbf{A} \breve{\boldsymbol{\theta}}-\mathbf{b})^{T} \boldsymbol{\Psi}^{-1}(\mathbf{A} \breve{\boldsymbol{\theta}}-\mathbf{b})+\lambda\left(\mathbf{q}^{T} \breve{\boldsymbol{\theta}}+\breve{\boldsymbol{\theta}}^{T} \mathbf{P} \breve{\boldsymbol{\theta}}\right)
$$

where $\lambda$ is the corresponding Lagrange multiplier. The CWLS solution using the RSS measurements is given by (see Appen$\operatorname{dix}$ A.2)

$$
\widehat{\boldsymbol{\theta}}=\left(\mathbf{A}^{T} \boldsymbol{\Psi}^{-1} \mathbf{A}+\lambda \mathbf{P}\right)^{-1}\left(\mathbf{A}^{T} \boldsymbol{\Psi}^{-1} \mathbf{b}-\frac{\lambda}{2} \mathbf{q}\right)
$$

where $\lambda$ is determined from the 5-root equation:

$$
\begin{aligned}
c_{3} f_{3} & -\frac{\lambda}{2} c_{3} g_{3}+\sum_{i=1}^{2} \frac{c_{i} f_{i}}{1+\lambda \gamma_{i}}-\frac{\lambda}{2} \sum_{i=1}^{2} \frac{c_{i} g_{i}}{1+\lambda \gamma_{i}}+\sum_{i=1}^{2} \frac{e_{i} f_{i} \gamma_{i}}{\left(1+\lambda \gamma_{i}\right)^{2}} \\
& -\frac{\lambda}{2} \sum_{i=1}^{2} \frac{e_{i} g_{i} \gamma_{i}}{\left(1+\lambda \gamma_{i}\right)^{2}}-\frac{\lambda}{2} \sum_{i=1}^{2} \frac{c_{i} f_{i} \gamma_{i}}{\left(1+\lambda \gamma_{i}\right)^{2}}+\frac{\lambda^{2}}{4} \sum_{i=1}^{2} \frac{c_{i} g_{i} \gamma_{i}}{\left(1+\lambda \gamma_{i}\right)^{2}}=0 .
\end{aligned}
$$

The $\left\{c_{i}\right\},\left\{e_{i}\right\},\left\{f_{i}\right\}$, and $\left\{g_{i}\right\}, i=1,2,3$, have been defined in Appendix A.2. The CWLS solution using the RSS measurements is found by the following procedure.

(i) Obtain the real roots of (46) using a root finding algorithm.

(ii) Put the real $\lambda$ 's back to (45) and obtain subestimates of $\hat{\boldsymbol{\theta}}$.

(iii) The subestimate that yields the smallest objective value of $(\mathbf{A} \breve{\boldsymbol{\theta}}-\mathbf{b})^{T} \Psi^{-1}(\mathbf{A} \breve{\boldsymbol{\theta}}-\mathbf{b})$ is taken as the globally optimal CWLS solution. 


\subsection{TOA [20]}

Since the models of the TOA and RSS will have the same form if the propagation constant is equal to unity, putting $a=1$ in Section 3.2 yields the algorithm of the CWLS estimator using the TOA data.

\subsection{AOA}

In the absence of noise, (13) becomes

$$
\begin{aligned}
\tan \left(r_{\mathrm{AOA}, i}\right) & =\frac{\sin \left(r_{\mathrm{AOA}, i}\right)}{\cos \left(r_{\mathrm{AOA}, i}\right)} \\
& =\frac{y-y_{i}}{x-x_{i}}, \quad i=1,2, \ldots, M .
\end{aligned}
$$

By cross-multiplying and rearranging (47), a set of linear equations in $x$ and $y$ for the AOA measurements is obtained as

$$
\begin{aligned}
& x \sin \left(r_{\mathrm{AOA}, i}\right)-y \cos \left(r_{\mathrm{AOA}, i}\right) \\
& \quad=x_{i} \sin \left(r_{\mathrm{AOA}, i}\right)-y_{i} \cos \left(r_{\mathrm{AOA}, i}\right), \quad i=1,2, \ldots, M .
\end{aligned}
$$

Expressing (48) in matrix form, we have [16]

$$
\mathbf{H x}=\mathbf{k},
$$

where

$$
\begin{gathered}
\mathbf{H}=\left[\begin{array}{cc}
\sin \left(r_{\mathrm{AOA}, 1}\right) & -\cos \left(r_{\mathrm{AOA}, 1}\right) \\
\vdots & \vdots \\
\sin \left(r_{\mathrm{AOA}, M}\right) & -\cos \left(r_{\mathrm{AOA}, M}\right)
\end{array}\right], \\
\mathbf{k}=\left[\begin{array}{c}
x_{1} \sin \left(r_{\mathrm{AOA}, 1}\right)-y_{1} \cos \left(r_{\mathrm{AOA}, 1}\right) \\
\vdots \\
x_{M} \sin \left(r_{\mathrm{AOA}, M}\right)-y_{M} \cos \left(r_{\mathrm{AOA}, M}\right)
\end{array}\right] .
\end{gathered}
$$

To improve the performance of the LS estimator of [16], we propose to use WLS to estimate the MS location $\mathbf{x}$ and the solution is

$$
\begin{aligned}
\widehat{\mathbf{x}} & =\arg \min _{\breve{\mathbf{x}}}(\mathbf{H} \breve{\mathbf{x}}-\mathbf{k})^{T} \boldsymbol{\Omega}^{-1}(\mathbf{H} \breve{\mathbf{x}}-\mathbf{k}) \\
& =\left(\mathbf{H}^{T} \boldsymbol{\Omega}^{-1} \mathbf{H}\right)^{-1} \mathbf{H}^{T} \boldsymbol{\Omega}^{-1} \mathbf{k},
\end{aligned}
$$

where $\boldsymbol{\Omega}^{-1}$ is the corresponding weighting matrix and $\breve{\mathbf{x}}=$ $[\breve{x}, \breve{y}]^{T}$. Again, we use the BLUE technique to determine the optimum $\Omega$ as follows. In the presence of measurement errors, (48) becomes

$$
\begin{aligned}
& x \sin \left(\phi_{i}+n_{\mathrm{AOA}, i}\right)-y \cos \left(\phi_{i}+n_{\mathrm{AOA}, i}\right) \\
& =x_{i} \sin \left(\phi_{i}+n_{\mathrm{AOA}, i}\right)-y_{i} \cos \left(\phi_{i}+n_{\mathrm{AOA}, i}\right), \quad i=1,2, \ldots, M .
\end{aligned}
$$

It is noteworthy that (52) is similar to the Taylor series linearization based on a geometrical viewpoint [17], although the latter considers only one AOA measurement with the corresponding BS locates at the origin. By expanding $\sin \left(\phi_{i}+\right.$ $\left.n_{\mathrm{AOA}, i}\right)$ and $\cos \left(\phi_{i}+n_{\mathrm{AOA}, i}\right)$, and considering sufficiently small angle errors such that $\sin \left(n_{\mathrm{AOA}, i}\right) \approx n_{\mathrm{AOA}, i}$ and $\cos \left(n_{\mathrm{AOA}, i}\right) \approx$ 1 , we obtain the residual error in $r_{\mathrm{AOA}, i}$ as

$$
\begin{array}{r}
\delta_{i}=n_{\mathrm{AOA}, i}\left[\left(x-x_{i}\right) \cos \left(\phi_{i}\right)+\left(y-y_{i}\right) \sin \left(\phi_{i}\right)\right], \\
i=1,2, \ldots, M .
\end{array}
$$

In vector form, $\left\{\delta_{i}\right\}$ is expressed as

$$
\boldsymbol{\delta}=\left[\begin{array}{c}
n_{\mathrm{AOA}, 1}\left[\left(x-x_{1}\right) \cos \left(\phi_{1}\right)+\left(y-y_{1}\right) \sin \left(\phi_{1}\right)\right] \\
n_{\mathrm{AOA}, 2}\left[\left(x-x_{2}\right) \cos \left(\phi_{2}\right)+\left(y-y_{2}\right) \sin \left(\phi_{2}\right)\right] \\
\vdots \\
n_{\mathrm{AOA}, M}\left[\left(x-x_{M}\right) \cos \left(\phi_{M}\right)+\left(y-y_{M}\right) \sin \left(\phi_{M}\right)\right]
\end{array}\right] .
$$

Thus the inverse of the optimum weighting matrix, $\boldsymbol{\Omega}^{\circ}$, is

$$
\boldsymbol{\Omega}^{o}=E\left\{\boldsymbol{\delta} \boldsymbol{\delta}^{T}\right\}=\mathbf{s}_{3} \mathbf{s}_{3}^{T} \odot \mathbf{C}_{\mathbf{n}, \mathrm{AOA}},
$$

where

$$
\mathbf{s}_{3}=\left[\begin{array}{c}
\left(x-x_{1}\right) \cos \left(\phi_{1}\right)+\left(y-y_{1}\right) \sin \left(\phi_{1}\right) \\
\left(x-x_{2}\right) \cos \left(\phi_{2}\right)+\left(y-y_{2}\right) \sin \left(\phi_{2}\right) \\
\vdots \\
\left(x-x_{M}\right) \cos \left(\phi_{M}\right)+\left(y-y_{M}\right) \sin \left(\phi_{M}\right)
\end{array}\right]=\left[\begin{array}{c}
d_{1} \\
d_{2} \\
\vdots \\
d_{M}
\end{array}\right]
$$

because $\cos \left(\phi_{i}\right)=\left(x-x_{i}\right) / d_{i}$ and $\sin \left(\phi_{i}\right)=\left(y-y_{i}\right) / d_{i}$. Again, since $\mathbf{s}_{3}$ involves the unknown parameters $\mathbf{x}$ and $\left\{\phi_{i}\right\}$, they will be approximated as $\hat{\mathbf{x}}$ and $\left\{r_{\mathrm{AOA}, i}\right\}$, respectively, in the actual implementation. In summary, the WLS procedure for AOA-based location is

(i) set $\mathbf{\Omega}=\mathbf{I}_{M}$;

(ii) use (51) to determine the estimate of $\mathbf{x}$;

(iii) construct $\boldsymbol{\Omega}$ based on (55) using the computed $\hat{\mathbf{x}}$ in step (ii) and repeat step (ii) until parameter convergence.

It is noteworthy that since $\mathbf{H}$ also consists of noise, we have already attempted to introduce constraints in the WLS solution in order to remove the bias due to the noisy components, but improvement over the WLS estimator has not been observed. As a result, it is believed that the noise in $\mathbf{H}$ can be ignored for sufficiently high signal-to-noise ratio (SNR) conditions. In fact, Pages-Zamora et al. [16] have similarly observed that the LS estimator performs even better than its total least squares counterpart. 


\subsection{TDOA-AOA hybrid}

It is apparent that combining different types of the measurements, if available, can improve location performance and/or reduce the number of receiving BSs. Among various hybrid schemes, the most popular one is to use the TDOA and AOA measurements simultaneously [17]. To perform TDOA-AOA mobile positioning, (48) is now rewritten by adding $y_{1} \cos \left(r_{\mathrm{AOA}, i}\right)-x_{1} \sin \left(r_{\mathrm{AOA}, i}\right)$ on both sides:

$$
\begin{array}{r}
\left(x-x_{1}\right) \sin \left(r_{\mathrm{AOA}, i}\right)-\left(y-y_{1}\right) \cos \left(r_{\mathrm{AOA}, i}\right) \\
=\left(x_{i}-x_{1}\right) \sin \left(r_{\mathrm{AOA}, i}\right)-\left(y_{i}-y_{1}\right) \cos \left(r_{\mathrm{AOA}, i}\right), \\
i=1,2, \ldots, M .
\end{array}
$$

Combining (19) and (57) into a single matrix-vector form yields

$$
\mathbf{B \vartheta}=\mathbf{w}
$$

where

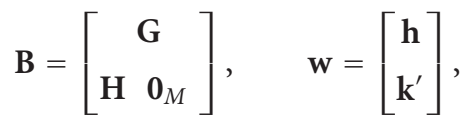

$$
\begin{aligned}
& \mathbf{k}^{\prime}=\left[\begin{array}{c}
0 \\
\left(x_{2}-x_{1}\right) \sin \left(r_{\mathrm{AOA}, 2}\right)-\left(y_{2}-y_{1}\right) \cos \left(r_{\mathrm{AOA}, 2}\right) \\
\vdots \\
\left(x_{M}-x_{1}\right) \sin \left(r_{\mathrm{AOA}, M}\right)-\left(y_{M}-y_{1}\right) \cos \left(r_{\mathrm{AOA}, M}\right)
\end{array}\right]
\end{aligned}
$$

with $\mathbf{0}_{M}$ is an $M \times 1$ column vector with all zeros. Then $\boldsymbol{\vartheta}$ is solved by minimizing

$$
(\mathbf{B \mathfrak { \vartheta }}-\mathbf{w})^{T} \mathbf{W}^{-1}(\mathbf{B} \breve{\vartheta}-\mathbf{w})
$$

subject to

$$
\breve{\boldsymbol{\vartheta}}^{T} \boldsymbol{\Sigma} \breve{\boldsymbol{\vartheta}}=0 .
$$

The optimum weighting matrix, denoted by $\mathbf{W}^{o^{-1}}$, is determined from the inverse of

$$
\mathbf{W}^{o}=\mathbf{s}_{4} \mathbf{s}_{4}^{T} \odot \mathbf{C}_{\mathbf{n}, \text { TDOA-AOA, }}
$$

where $\mathbf{s}_{4}=\left[\begin{array}{ll}\mathbf{s}_{1} & \mathbf{s}_{3}\end{array}\right]^{T}$ and $\mathbf{C}_{\mathbf{n}, \mathrm{TDOA}-\mathrm{AOA}}$ is the covariance matrix of the TDOA and AOA measurement errors. By following the estimation procedure in Section 3.1, the parameter vector $\hat{\boldsymbol{\vartheta}}$ is determined. Similarly, mobile location algorithms using AOA and RSS or TOA measurements can be deduced.
For TDOA-TOA or TDOA-RSS hybrid positioning, a simple and effective way is to convert the TOA and RSS, respectively, into TDOA measurements and then apply the CWLS TDOA-based location algorithm. Finally, it is straightforward to combine TOA and RSS measurements via converting the former to the latter or vice versa. Localization with more than two types of measurements can be extended easily in a similar manner.

\section{PERFORMANCE ANALYSIS}

As briefly mentioned in Section 1, the CWLS and WLS estimators in Section 3 can achieve zero bias and the CRLB approximately when the noise is uncorrelated and small in power. In the following subsections we provide the proofs of this desirable property for each measurement case.

\subsection{Mean and variance analysis for generic unconstrained minimization problems}

The idea behind the performance analysis here is to recast the CWLS estimators to unconstrained minimization problems, and then to use the analysis technique for unconstrained problems [28] to find out the mean and covariance of the estimators. To describe the latter, consider a generic unconstrained estimation problem as follows:

$$
\hat{\mathbf{y}}=\arg \min _{\breve{\mathbf{y}}} J(\breve{\mathbf{y}})
$$

where $J(\breve{\mathbf{y}})$ is a function continuous in $\breve{\mathbf{y}}$. Given that $\mathbf{y}$ is the true value of the estimated parameter, it is shown [28] that

$$
\begin{aligned}
\operatorname{bias}(\hat{\mathbf{y}}) & \approx-\left.E\left[\frac{\partial^{2} J}{\partial \breve{\mathbf{y}} \partial \breve{\mathbf{y}}^{T}}\right]^{-1} E\left[\frac{\partial J}{\partial \breve{\mathbf{y}}}\right]\right|_{\breve{\mathbf{y}}=\mathbf{y}}, \\
\mathbf{C}_{\mathbf{y}} & \left.\approx E\left[\frac{\partial^{2} J}{\partial \breve{\mathbf{y}} \partial \breve{\mathbf{y}}^{T}}\right]^{-1} E\left[\left(\frac{\partial J}{\partial \breve{\mathbf{y}}}\right)\left(\frac{\partial J}{\partial \breve{\mathbf{y}}}\right)^{T}\right] E\left[\frac{\partial^{2} J}{\partial \breve{\mathbf{y}}^{2} \breve{\mathbf{y}}^{T}}\right]^{-1}\right|_{\breve{\mathbf{y}}=\mathbf{y}},
\end{aligned}
$$

where bias $(\hat{\mathbf{y}})$ and $\mathbf{C}_{\mathbf{y}}$ represent the bias and the covariance matrix associated with $\hat{\mathbf{y}}$, respectively. The approximations in (64) and (65) are based on the assumption that noise variances are sufficiently small. In the following, we will apply (64) and (65) to show that all the developed algorithms are approximately unbiased and to produce their theoretical variances.

\subsection{TDOA}

Although the CWLS problem of (24) subject to (23) consists of a parameter vector $\breve{\vartheta}$ with 3 variables, namely, $\breve{x}-x_{1}, \breve{y}-y_{1}$, and $\breve{R}_{1}$, it can be reduced to a 2 -variable optimization problem using the relation of (18), that is, setting $\breve{R}_{1}=\left(\breve{\vartheta}_{1}^{T} \breve{\vartheta}_{1}\right)^{1 / 2}$ where $\breve{\vartheta}_{1}=\left[\begin{array}{ll}\breve{x}-x_{1} & \breve{y}-y_{1}\end{array}\right]^{T}$. In so doing, the CWLS position estimate using the TDOA measurements is equivalent 
to

$$
\widehat{\boldsymbol{\vartheta}}_{1}=\arg \min _{\breve{\vartheta}_{1}} J_{\mathrm{TDOA}}\left(\breve{\boldsymbol{\vartheta}}_{1}\right)
$$

where

$$
\begin{aligned}
J_{\mathrm{TDOA}}\left(\breve{\boldsymbol{\vartheta}}_{1}\right)= & {\left[\mathbf{S} \breve{\boldsymbol{\vartheta}}_{1}+\left(\breve{\boldsymbol{\vartheta}}_{1}^{T} \breve{\boldsymbol{\vartheta}}_{1}\right)^{1 / 2} \mathbf{r}_{\mathrm{TDOA}}-\mathbf{h}\right]^{T} } \\
& \times \mathbf{Y}^{-1}\left[\mathbf{S} \breve{\boldsymbol{\vartheta}}_{1}+\left(\breve{\boldsymbol{\vartheta}}_{1}^{T} \breve{\boldsymbol{\vartheta}}_{1}\right)^{1 / 2} \mathbf{r}_{\mathrm{TDOA}}-\mathbf{h}\right]
\end{aligned}
$$

which is the cost function of the CWLS algorithm using TDOA measurements in terms of $\breve{\vartheta}_{1}$ with

$$
\mathbf{S}=\left[\begin{array}{cc}
x_{2}-x_{1} & y_{2}-y_{1} \\
x_{3}-x_{1} & y_{3}-y_{1} \\
\vdots & \vdots \\
x_{M}-x_{1} & y_{M}-y_{1}
\end{array}\right]
$$

The values of $E\left[\partial J_{\mathrm{TDOA}}\left(\breve{\boldsymbol{\vartheta}}_{1}\right) / \partial \breve{\boldsymbol{\vartheta}}_{1}\right], E\left[\partial^{2} J_{\mathrm{TDOA}}\left(\breve{\boldsymbol{\vartheta}}_{1}\right) / \partial \breve{\boldsymbol{\vartheta}}_{1} \partial \breve{\boldsymbol{\vartheta}}_{1}^{T}\right]$, and $E\left[\left(\partial J_{\mathrm{TDOA}}\left(\breve{\vartheta}_{1}\right) / \partial \breve{\vartheta}_{1}\right)\left(\partial J_{\mathrm{TDOA}}\left(\breve{\vartheta}_{1}\right) / \partial \breve{\vartheta}_{1}\right)^{T}\right]$ at $\breve{\vartheta}_{1}=\boldsymbol{\vartheta}_{1}$ are calculated in Appendix B.1. Using (64) and (65) with $J=$ $J_{\text {TDOA }}\left(\breve{\vartheta}_{1}\right)$, the mean and the covariance matrix of the MS position estimated by the CWLS algorithm are

$$
\begin{gathered}
E[\hat{\mathbf{x}}] \approx \mathbf{x}, \\
\mathbf{C}_{\mathbf{x}} \approx\left\{\left[\mathbf{S}^{T}+d_{1}^{-1}\left(\mathbf{x}-\mathbf{x}_{1}\right)\left(\mathbf{s}_{1}^{T}-d_{1} \mathbf{1}_{M-1}^{T}\right)\right],\right. \\
\left.\times \mathbf{Y}^{-1}\left[\mathbf{S}+d_{1}^{-1}\left(\mathbf{s}_{1}-d_{1} \mathbf{1}_{M-1}\right)\left(\mathbf{x}-\mathbf{x}_{1}\right)^{T}\right]\right\}^{-1},
\end{gathered}
$$

where $\mathbf{1}_{M-1}$ is denoted as an $(M-1) \times 1$ column vector with all ones. Equation (69) shows that the estimator is approximately unbiased, while the two diagonal elements in (70) correspond to the variance of the position estimate $\hat{\mathbf{x}}$. Now we are going to compute $\mathbf{C}_{\mathbf{x}}$ particularly when all the measurement errors are uncorrelated. This implies that the covariance matrix for the TDOA measurement errors has the form of

$$
\mathbf{C}_{\mathbf{n}, \mathrm{TDOA}}=\left[\begin{array}{cccc}
\sigma_{\mathrm{TDOA}, 2}^{2} & 0 & \cdots & 0 \\
0 & \sigma_{\mathrm{TDOA}, 3}^{2} & \cdots & 0 \\
\vdots & \vdots & \ddots & \vdots \\
0 & 0 & \cdots & \sigma_{\mathrm{TDOA}, M}^{2}
\end{array}\right]
$$

Considering sufficiently small error conditions such that $\Upsilon \approx$ $\Upsilon^{o}$, we have

$$
\begin{aligned}
\mathbf{Y} & \approx \mathbf{s}_{1} \mathbf{s}_{1}^{T} \odot \mathbf{C}_{\mathbf{n}, \mathrm{TDOA}} \\
& =\left[\begin{array}{cccc}
d_{2}^{2} \sigma_{\mathrm{TDOA}, 2}^{2} & 0 & \cdots & 0 \\
0 & d_{3}^{2} \sigma_{\mathrm{TDOA}, 3}^{2} & \cdots & 0 \\
\vdots & \vdots & \ddots & \vdots \\
0 & 0 & \cdots & d_{M}^{2} \sigma_{\mathrm{TDOA}, M}^{2}
\end{array}\right]
\end{aligned}
$$

We also note that

$$
\left[\mathbf{S}^{T}+d_{1}^{-1}\left(\mathbf{x}-\mathbf{x}_{1}\right)\left(\mathbf{s}_{1}^{T}-d_{1} \mathbf{1}_{M-1}^{T}\right)\right]=\left[\begin{array}{lll}
\left(x_{2}-x_{1}\right)+\left(x-x_{1}\right) \frac{d_{2}-d_{1}}{d_{1}} & \cdots & \left(x_{M}-x_{1}\right)+\left(x-x_{1}\right) \frac{d_{M}-d_{1}}{d_{1}} \\
\left(y_{2}-y_{1}\right)+\left(y-y_{1}\right) \frac{d_{2}-d_{1}}{d_{1}} & \cdots & \left(y_{M}-y_{1}\right)+\left(y-y_{1}\right) \frac{d_{M}-d_{1}}{d_{1}}
\end{array}\right]
$$

and $\left[\mathbf{S}+d_{1}^{-1}\left(\mathbf{s}_{1}-d_{1} \mathbf{1}_{M-1}\right)\left(\mathbf{x}-\mathbf{x}_{1}\right)^{T}\right]$ is given by the transpose of (73).
Substituting (72) and (73) into (70), the inverse of covariance matrix $\mathbf{C}_{\mathbf{x}}$ is calculated as

$$
\mathbf{C}_{\mathbf{x}}^{-1} \approx\left[\begin{array}{cc}
\sum_{i=2}^{M} \frac{1}{\sigma_{\mathrm{TDOA}, i}^{2}}\left[\frac{x-x_{i}}{d_{i}}-\frac{x-x_{1}}{d_{1}}\right]^{2} & \sum_{i=2}^{M} \frac{1}{\sigma_{\mathrm{TDOA}, i}^{2}}\left[\frac{x-x_{i}}{d_{i}}-\frac{x-x_{1}}{d_{1}}\right]\left[\frac{y-y_{i}}{d_{i}}-\frac{y-y_{1}}{d_{1}}\right] \\
\sum_{i=2}^{M} \frac{1}{\sigma_{\mathrm{TDOA}, i}^{2}}\left[\frac{x-x_{i}}{d_{i}}-\frac{x-x_{1}}{d_{1}}\right]\left[\frac{y-y_{i}}{d_{i}}-\frac{y-y_{1}}{d_{1}}\right] & \sum_{i=2}^{M} \frac{1}{\sigma_{\mathrm{TDOA}, i}^{2}}\left[\frac{y-y_{i}}{d_{i}}-\frac{y-y_{1}}{d_{1}}\right]^{2}
\end{array}\right] .
$$


On the other hand, the Fisher information matrix (FIM) for the TDOA-based mobile location problem with uncorrelated measurement errors is computed in Appendix C as shown below

$$
\mathbf{I}_{\mathrm{TDOA}}(\mathbf{x})=\left[\begin{array}{c}
\left.\sum_{i=2}^{M} \frac{1}{\sigma_{\mathrm{TDOA}, i}^{2}}\left[\frac{x-x_{i}}{d_{i}}-\frac{x-x_{1}}{d_{1}}\right]^{2} \sum_{i=2}^{M} \frac{1}{\sigma_{\mathrm{TDOA}, i}^{2}}\left[\frac{x-x_{i}}{d_{i}}-\frac{x-x_{1}}{d_{1}}\right]\left[\frac{y-y_{i}}{d_{i}}-\frac{y-y_{1}}{d_{1}}\right]\right] \\
\sum_{i=2}^{M} \frac{1}{\sigma_{\mathrm{TDOA}, i}^{2}}\left[\frac{x-x_{i}}{d_{i}}-\frac{x-x_{1}}{d_{1}}\right]\left[\frac{y-y_{i}}{d_{i}}-\frac{y-y_{1}}{d_{1}}\right] \\
\sum_{i=2}^{M} \frac{1}{\sigma_{\mathrm{TDOA}, i}^{2}}\left[\frac{y-y_{i}}{d_{i}}-\frac{y-y_{1}}{d_{1}}\right]^{2}
\end{array}\right]
$$

which implies $\mathbf{C}_{\mathbf{x}}^{-1} \approx \mathbf{I}_{\mathrm{TDOA}}(\mathbf{x})$. As a result, the performance of the TDOA-based mobile positioning algorithm via the use of CWLS achieves the CRLB for uncorrelated measurement errors. It is also expected that the optimality still holds when the TDOA measurement errors are correlated.

\subsection{RSS}

Similar to Section 4.1, $\breve{R}_{2}$ in $\breve{\boldsymbol{\theta}}$ is substituted by $\mathbf{x}^{T} \mathbf{x}$ so the CWLS solution using the RSS measurements is equivalent to

$$
\widehat{\mathbf{x}}=\arg \min _{\breve{\mathbf{x}}} J_{\mathrm{RSS}}(\breve{\mathbf{x}}),
$$

where

$$
\begin{aligned}
J_{\mathrm{RSS}}(\breve{\mathbf{x}})= & {\left[\mathbf{X}_{\mathrm{BS}} \breve{\mathbf{x}}-0.5\left(\breve{\mathbf{x}}^{T} \breve{\mathbf{x}}\right) \mathbf{1}_{M}-\mathbf{b}\right]^{T} } \\
& \times \boldsymbol{\Psi}^{-1}\left[\mathbf{X}_{\mathrm{BS}} \breve{\mathbf{x}}-0.5\left(\breve{\mathbf{x}}^{T} \breve{\mathbf{x}}\right) \mathbf{1}_{M}-\mathbf{b}\right]
\end{aligned}
$$

with

$$
\mathbf{X}_{\mathrm{BS}}=\left[\begin{array}{cc}
x_{1} & y_{1} \\
x_{2} & y_{2} \\
\vdots & \vdots \\
x_{M} & y_{M}
\end{array}\right]
$$

The required values of the derivatives have been computed in Appendix B.2. Putting them into (64) and (65) with $J=$ $J_{\text {RSS }}(\breve{\mathbf{x}})$ gives

$$
\begin{gathered}
E[\hat{\mathbf{x}}] \approx \mathbf{x}, \\
\mathbf{C}_{\mathbf{x}} \approx\left\{\left(\mathbf{X}_{\mathrm{BS}}^{T}-\mathbf{x} \mathbf{1}_{M}^{T}\right) \boldsymbol{\Psi}^{-1}\left(\mathbf{X}_{\mathrm{BS}}-\mathbf{1}_{M} \mathbf{x}^{T}\right)\right\}^{-1} .
\end{gathered}
$$

Again, the unbiasedness of the algorithm is illustrated in (79). For uncorrelated measurement errors, we have

$$
\mathrm{C}_{\mathbf{n}, \mathrm{RSS}}=\left[\begin{array}{cccc}
\sigma_{\mathrm{RSS}, 1}^{2} & 0 & \cdots & 0 \\
0 & \sigma_{\mathrm{RSS}, 2}^{2} & \cdots & 0 \\
\vdots & \vdots & \ddots & \vdots \\
0 & 0 & \cdots & \sigma_{\mathrm{RSS}, M}^{2}
\end{array}\right]
$$

Assuming ideal weighting matrix as in the previous analysis, the inverse of $\Psi^{-1}$ for the RSS-based algorithm is

$$
\begin{aligned}
& \boldsymbol{\Psi} \approx \mathbf{s}_{2} \mathbf{s}_{2}^{T} \odot \mathbf{C}_{\mathbf{n}, \mathrm{RSS}} \\
& =\left[\begin{array}{cccc}
\frac{1}{a^{2}} d_{1}^{2(2-a)} \sigma_{\mathrm{RSS}, 1}^{2} & 0 & \cdots & 0 \\
0 & \frac{1}{a^{2}} d_{2}^{2(2-a)} \sigma_{\mathrm{RSS}, 2}^{2} & \cdots & 0 \\
\vdots & \vdots & \ddots & \vdots \\
0 & 0 & \cdots & \frac{1}{a^{2}} d_{M}^{2(2-a)} \sigma_{\mathrm{RSS}, M}^{2}
\end{array}\right] \text {. }
\end{aligned}
$$

It is also noted that

$$
\mathbf{X}_{\mathrm{BS}}^{T}-\mathbf{x} \mathbf{1}_{M}^{T}=\left[\begin{array}{llll}
x_{1}-x & x_{2}-x & \cdots & x_{M}-x \\
y_{1}-y & y_{2}-y & \cdots & y_{M}-y
\end{array}\right]
$$

and $\left(\mathbf{X}_{\mathrm{BS}}-\mathbf{1}_{M} \mathbf{x}^{T}\right)$ is the transpose of (83). Hence the inverse of the covariance matrix is 


$$
\mathbf{C}_{\mathbf{x}}^{-1} \approx\left(\mathbf{X}_{\mathrm{BS}}^{T}-\mathbf{x} \mathbf{1}_{M}^{T}\right) \boldsymbol{\Psi}^{-1}\left(\mathbf{X}_{\mathrm{BS}}-\mathbf{1}_{M} \mathbf{x}^{T}\right)=\left[\begin{array}{cc}
\sum_{i=1}^{M} \frac{a^{2}\left(x-x_{i}\right)^{2} d_{i}^{2(a-2)}}{\sigma_{\mathrm{RSS}, i}^{2}} & \sum_{i=1}^{M} \frac{a^{2}\left(x-x_{i}\right)\left(y-y_{i}\right) d_{i}^{2(a-2)}}{\sigma_{\mathrm{RSS}, i}^{2}} \\
\sum_{i=1}^{M} \frac{a^{2}\left(x-x_{i}\right)\left(y-y_{i}\right) d_{i}^{2(a-2)}}{\sigma_{\mathrm{RSS}, i}^{2}} & \sum_{i=1}^{M} \frac{a^{2}\left(y-y_{i}\right)^{2} d_{i}^{2(a-2)}}{\sigma_{\mathrm{RSS}, i}^{2}}
\end{array}\right] .
$$

From Appendix C, the FIM for RSS-based mobile location with uncorrelated measurement errors can be computed, which is given by

$\mathbf{I}_{\mathrm{RSS}}(\mathbf{x})$

$$
=\left[\begin{array}{cc}
\sum_{i=1}^{M} \frac{a^{2}\left(x-x_{i}\right)^{2} d_{i}^{2(a-2)}}{\sigma_{\mathrm{RSS}, i}^{2}} & \sum_{i=1}^{M} \frac{a^{2}\left(x-x_{i}\right)\left(y-y_{i}\right) d_{i}^{2(a-2)}}{\sigma_{\mathrm{RSS}, i}^{2}} \\
\sum_{i=1}^{M} \frac{a^{2}\left(x-x_{i}\right)\left(y-y_{i}\right) d_{i}^{2(a-2)}}{\sigma_{\mathrm{RSS}, i}^{2}} & \sum_{i=1}^{M} \frac{a^{2}\left(y-y_{i}\right)^{2} d_{i}^{2(a-2)}}{\sigma_{\mathrm{RSS}, i}^{2}}
\end{array}\right]
$$

which means $\mathbf{I}_{\mathrm{RSS}}(\mathbf{x}) \approx \mathbf{C}_{\mathbf{x}}^{-1}$, and thus the optimality of the RSS-based location algorithm for white disturbance is proved.

\subsection{TOA}

By putting $a=1$ in Section 4.2, the bias and variance expressions for the position estimate using the TOA data are obtained. Nevertheless, we have already shown that its estimation performance attains the CRLB in uncorrelated measurement errors in [20].

\section{5. $A O A$}

From Section 3.4, the WLS cost function for AOA-based mobile positioning is

$$
J_{\mathrm{AOA}}(\breve{\mathbf{x}})=(\mathbf{H} \breve{\mathbf{x}}-\mathbf{k})^{T} \boldsymbol{\Omega}^{-1}(\mathbf{H} \breve{\mathbf{x}}-\mathbf{k}) .
$$

In Appendix B.3, the mean and the covariance matrix of the MS position estimate are calculated as

$$
\begin{gathered}
E[\hat{\mathbf{x}}] \approx \mathbf{x}, \\
\mathrm{C}_{\mathbf{x}} \approx\left(\mathbf{H}^{T} \mathbf{\Omega}^{-1} \mathbf{H}\right)^{-1} .
\end{gathered}
$$

In particular, for uncorrelated measurement errors, $\mathbf{C}_{\mathbf{n}, \mathrm{AOA}}$ is of the form

$$
\mathbf{C}_{\mathbf{n}, \mathrm{AOA}}=\left[\begin{array}{cccc}
\sigma_{\mathrm{AOA}, 1}^{2} & 0 & \cdots & 0 \\
0 & \sigma_{\mathrm{AOA}, 2}^{2} & \cdots & 0 \\
\vdots & \vdots & \ddots & \vdots \\
0 & 0 & \cdots & \sigma_{\mathrm{AOA}, M}^{2}
\end{array}\right] .
$$

Considering sufficiently small noise conditions, we have

$$
\mathbf{\Omega} \approx \mathbf{s}_{3} \mathbf{s}_{3}^{T} \odot \mathbf{C}_{\mathbf{n}, \mathrm{AOA}}
$$

$$
=\left[\begin{array}{cccc}
d_{1}^{2} \sigma_{\mathrm{AOA}, 1}^{2} & 0 & \cdots & 0 \\
0 & d_{2}^{2} \sigma_{\mathrm{AOA}, 2}^{2} & \cdots & 0 \\
\vdots & \vdots & \ddots & \vdots \\
0 & 0 & \cdots & d_{M}^{2} \sigma_{\mathrm{AOA}, M}^{2}
\end{array}\right]
$$

$$
\mathbf{H} \approx\left[\begin{array}{cc}
\frac{y-y_{1}}{d_{1}} & -\frac{x-x_{1}}{d_{1}} \\
\frac{y-y_{2}}{d_{2}} & -\frac{x-x_{2}}{d_{2}} \\
\vdots & \vdots \\
\frac{y-y_{M}}{d_{M}} & -\frac{x-x_{M}}{d_{M}}
\end{array}\right] .
$$

Putting (90) into (88) yields

$$
\begin{aligned}
\mathbf{C}_{\mathbf{x}}^{-1} & \approx \mathbf{H}^{T} \mathbf{\Omega}^{-1} \mathbf{H} \\
& \approx\left[\begin{array}{cc}
\sum_{i=1}^{M} \frac{\left(y-y_{i}\right)^{2}}{\sigma_{\mathrm{AOA}, i}^{2} d_{i}^{4}} & -\sum_{i=1}^{M} \frac{\left(x-x_{i}\right)\left(y-y_{i}\right)}{\sigma_{\mathrm{AOA}, i}^{2} d_{i}^{4}} \\
-\sum_{i=1}^{M} \frac{\left(x-x_{i}\right)\left(y-y_{i}\right)}{\sigma_{\mathrm{AOA}, i}^{2} d_{i}^{4}} & \sum_{i=1}^{M} \frac{\left(x-x_{i}\right)^{2}}{\sigma_{\mathrm{AOA}, i}^{2} d_{i}^{4}}
\end{array}\right] .
\end{aligned}
$$

On the other hand, the FIM for AOA-based mobile location with uncorrelated measurement errors is computed in Appendix C as

$$
\mathbf{I}_{\mathrm{AOA}}(\mathbf{x})=\left[\begin{array}{cc}
\sum_{i=1}^{M} \frac{\left(y-y_{i}\right)^{2}}{\sigma_{\mathrm{AOA}, i}^{2} d_{i}^{4}} & -\sum_{i=1}^{M} \frac{\left(x-x_{i}\right)\left(y-y_{i}\right)}{\sigma_{\mathrm{AOA}, i}^{2} d_{i}^{4}} \\
-\sum_{i=1}^{M} \frac{\left(x-x_{i}\right)\left(y-y_{i}\right)}{\sigma_{\mathrm{AOA}, i}^{2} d_{i}^{4}} & \sum_{i=1}^{M} \frac{\left(x-x_{i}\right)^{2}}{\sigma_{\mathrm{AOA}, i}^{2} d_{i}^{4}}
\end{array}\right]
$$


which implies $\mathbf{I}_{\mathrm{AOA}}(\mathbf{x}) \approx \mathbf{C}_{\mathbf{x}}^{-1}$. As a result, the performance of using the WLS estimator for AOA-based mobile location with uncorrelated measurement errors is optimal under small noise conditions.

\subsection{TDOA-AOA hybrid}

Similar to Section 4.1, the CWLS position estimate using both TDOA and AOA measurements is equivalent to

$$
\widehat{\boldsymbol{\vartheta}}_{1}=\arg \min _{\breve{\mathfrak{\vartheta}}_{1}} J_{\text {TDOA-AOA }}\left(\breve{\vartheta}_{1}\right)
$$

where

\section{$J_{\mathrm{TDOA}-\mathrm{AOA}}\left(\breve{\vartheta}_{1}\right)$}

$$
\begin{aligned}
= & (\mathbf{B} \breve{\boldsymbol{\vartheta}}-\mathbf{w})^{T} \mathbf{W}^{-1}(\mathbf{B} \breve{\boldsymbol{\vartheta}}-\mathbf{w}) \\
= & {\left[\left[\begin{array}{c}
\mathbf{S} \\
\mathbf{H}
\end{array}\right] \breve{\boldsymbol{\vartheta}}_{1}+\left(\breve{\boldsymbol{\vartheta}}_{1}^{T} \breve{\boldsymbol{\vartheta}}_{1}\right)^{1 / 2}\left[\begin{array}{c}
\mathbf{r}_{\mathrm{TDOA}} \\
\mathbf{0}_{M}
\end{array}\right]-\mathbf{w}\right]^{T} } \\
& \times \mathbf{W}^{-1}\left[\left[\begin{array}{c}
\mathbf{S} \\
\mathbf{H}
\end{array}\right] \breve{\mathfrak{\vartheta}}_{1}+\left(\breve{\boldsymbol{\vartheta}}_{1}^{T} \breve{\mathfrak{\vartheta}}_{1}\right)^{1 / 2}\left[\begin{array}{c}
\mathbf{r}_{\mathrm{TDOA}} \\
\mathbf{0}_{M}
\end{array}\right]-\mathbf{w}\right]
\end{aligned}
$$

with

$$
\mathbf{B}=\left[\begin{array}{cc}
\mathbf{S} & \mathbf{r}_{\mathrm{TDOA}} \\
\mathbf{H} & \mathbf{0}_{M}
\end{array}\right] \text {. }
$$

In Appendix B.4, we have shown that

$$
E[\widehat{\mathbf{x}}] \approx \mathbf{x}
$$

which indicates its unbiasedness and

$$
\begin{aligned}
\mathbf{C}_{\mathbf{x}} \approx & \left\{\left[\left[\begin{array}{ll}
\mathbf{S}^{T} & \mathbf{H}^{T}
\end{array}\right]+\left(\mathbf{x}-\mathbf{x}_{1}\right)\left(d_{1}^{-1}\left[\begin{array}{c}
\mathbf{s}_{1} \\
\mathbf{0}_{M}
\end{array}\right]-\left[\begin{array}{c}
\mathbf{1}_{M-1} \\
\mathbf{0}_{M}
\end{array}\right]\right)^{T}\right]\right. \\
& \left.\times \mathbf{W}^{-1}\left[\left[\begin{array}{l}
\mathbf{S} \\
\mathbf{H}
\end{array}\right]+\left(d_{1}^{-1}\left[\begin{array}{c}
\mathbf{s}_{1} \\
\mathbf{0}_{M}
\end{array}\right]-\left[\begin{array}{c}
\mathbf{1}_{M-1} \\
\mathbf{0}_{M}
\end{array}\right]\right)\left(\mathbf{x}-\mathbf{x}_{1}\right)^{T}\right]\right\}^{-1} \\
= & \left\{\left[\mathbf{S}^{T}+d_{1}^{-1}\left(\mathbf{x}-\mathbf{x}_{1}\right)\left(\mathbf{s}_{1}^{T}-d_{1} \mathbf{1}_{M-1}^{T}\right) \mathbf{H}^{T}\right]\right. \\
& \times \mathbf{W}^{-1}\left[\begin{array}{c}
\left.\left.\mathbf{S}+d_{1}^{-1}\left(\mathbf{s}_{1}-d_{1} \mathbf{1}_{M-1}\right)\left(\mathbf{x}-\mathbf{x}_{1}\right)^{T}\right]\right\}^{-1} . \\
\mathbf{H}
\end{array}\right.
\end{aligned}
$$

In particular, for uncorrelated measurement errors, we have

$$
\mathbf{C}_{\mathbf{n}, \mathrm{TDOA}-\mathrm{AOA}}=\left[\begin{array}{cc}
\mathbf{C}_{\mathbf{n}, \mathrm{TDOA}} & \mathbf{O}_{(M-1) \times M} \\
\mathbf{O}_{M \times(M-1)} & \mathbf{C}_{\mathbf{n}, \mathrm{AOA}}
\end{array}\right],
$$

where

$$
\begin{aligned}
\mathbf{C}_{\mathbf{n}, \mathrm{TDOA}} & =\operatorname{diag}\left(\sigma_{\mathrm{TDOA}, 2}^{2}, \sigma_{\mathrm{TDOA}, 3}^{2}, \ldots, \sigma_{\mathrm{TDOA}, M}^{2}\right), \\
\mathbf{C}_{\mathbf{n}, \mathrm{AOA}} & =\operatorname{diag}\left(\sigma_{\mathrm{AOA}, 1}^{2}, \sigma_{\mathrm{AOA}, 2}^{2}, \ldots, \sigma_{\mathrm{AOA}, M}^{2}\right),
\end{aligned}
$$

and $\mathbf{O}_{(M-1) \times M}$ is denoted as an $(M-1) \times M$ matrix with all zeros. Using the ideal weighting matrix, we get

$$
\begin{aligned}
\mathbf{W} & =\mathbf{s}_{4} \mathbf{s}_{4}^{T} \odot \mathbf{C}_{\mathbf{n}, \mathrm{TDOA}-\mathrm{AOA}} \\
& =\left[\begin{array}{cc}
\mathbf{s}_{1} \mathbf{s}_{1}^{T} \odot \mathbf{C}_{\mathbf{n}, \mathrm{TDOA}} & \mathbf{O}_{(M-1) \times M} \\
\mathbf{O}_{M \times(M-1)} & \mathbf{s}_{3} \mathbf{s}_{3}^{T} \odot \mathbf{C}_{\mathbf{n}, \mathrm{AOA}}
\end{array}\right] \\
& =\left[\begin{array}{cc}
\mathbf{Y} & \mathbf{O}_{(M-1) \times M} \\
\mathbf{O}_{M \times(M-1)} & \boldsymbol{\Omega}
\end{array}\right]
\end{aligned}
$$

which is a diagonal matrix. Substituting (100) into (97) yields

$$
\mathbf{C}_{\mathbf{x}}^{-1} \approx\left[\mathbf{S}^{T}+d_{1}^{-1}\left(\mathbf{x}-\mathbf{x}_{1}\right)\left(\mathbf{s}_{1}^{T}-d_{1} \mathbf{1}_{M-1}^{T}\right)\right]
$$

$$
\begin{aligned}
& \times \mathbf{\Upsilon}^{-1}\left[\mathbf{S}+d_{1}^{-1}\left(\mathbf{s}_{1}-d_{1} \mathbf{1}_{M-1}\right)\left(\mathbf{x}-\mathbf{x}_{1}\right)^{T}\right] \\
& +\mathbf{H}^{T} \mathbf{\Omega}^{-1} \mathbf{H} .
\end{aligned}
$$

In Appendix C, the FIM for the TDOA-AOA hybrid mobile positioning problem with uncorrelated errors can be computed as

$$
\mathbf{I}_{\mathrm{TDOA}-\mathrm{AOA}}(\mathbf{x})=\mathbf{I}_{\mathrm{TDOA}}(\mathbf{x})+\mathbf{I}_{\mathrm{AOA}}(\mathbf{x}) .
$$

From the results of (74), (75), (91), and (92), it is noted that $\mathbf{C}_{\mathbf{x}}^{-1} \approx \mathbf{I}_{\mathrm{TDOA}-\mathrm{AOA}}$. As a result, it is proved that the performance of the TDOA-AOA hybrid mobile positioning algorithm achieves the CRLB for sufficiently small uncorrelated noise conditions.

\section{SIMULATION RESULTS}

Computer simulation using MATLAB had been conducted to evaluate the performance of the proposed TOA-based, TDOA-based, RSS-based, AOA-based, and TDOA-AOA hybrid mobile positioning algorithms. Comparisons with the NLS approach as well as corresponding CRLBs were also made. We considered a 5-BS geometry with coordinates $[0,0] \mathrm{m},[3000 \sqrt{3}, 3000] \mathrm{m},[0,6000] \mathrm{m},[-3000 \sqrt{3}$, $3000] \mathrm{m}$, and $[-3000 \sqrt{3},-3000] \mathrm{m}$, while the MS position was fixed at $[x, y]=[1000,2000] \mathrm{m}$. The value of $a$ was set 


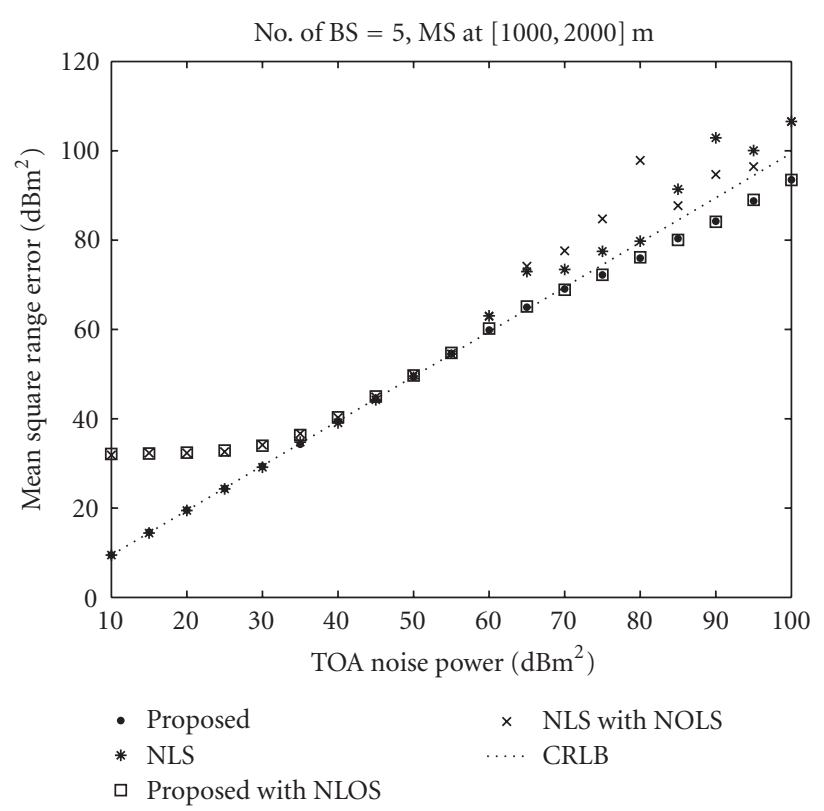

Figure 1: Mean square range errors for TOA measurements in uncorrelated noise.

to be 2 in all RSS measurements. For the proposed approach, steps (ii) and (iii) of the TDOA-based and TDOA-AOA hybrid algorithms and step (ii) of the AOA-based algorithm were only repeated once because no obvious improvement was observed for more iterations. On the other hand, we used the Newton-Raphson iterative procedure in the NLS implementation with three iterations. For TDOA, TOA, and RSS measurements, NLS initialization was given by (28) and (45) with setting the values of the Lagrange multipliers to zero. As for AOA measurements, (51) was employed to initialize the NLS estimator with $\boldsymbol{\Omega}=\mathbf{I}_{M}$. All results were averages of 1000 independent runs.

Figure 1 shows the mean square range errors (MSREs) of the TOA-based CWLS and NLS estimators as well as CRLB versus power of distance error based on the TOA measurements. For simplicity, we assumed that the disturbances in the TOA measurements, namely, $\left\{n_{\mathrm{TOA}, i}\right\}$, were white Gaussian processes with identical variances. The MSRE was defined as $E\left[(x-\hat{x})^{2}+(y-\hat{y})^{2}\right]$ and its unit was $\mathrm{m}^{2}$, which became $\mathrm{dBm}^{2}$ in $\mathrm{dB}$ scale. We observe that the performance of the proposed and NLS methods met the CRLB when the TOA noise power was less than $75 \mathrm{dBm}^{2}$ and $60 \mathrm{dBm}^{2}$, respectively, which indicated that the former had a larger optimum operation range. The effect of positive mean TOA errors, which corresponded to NLOS propagation, was also illustrated in the same figure. Here the range measurements were modeled as

$$
r_{\mathrm{TOA}, i}=d_{i}+n_{\mathrm{TOA}, i}+N u_{i}
$$

where $N=100 \mathrm{~m}$ was the maximum error introduced by NLOS and $u_{i}, i=1,2, \ldots, M$, were independent uniformly distributed random numbers ranged from 0 to 1 . It is seen that the nonzero mean errors introduced biases in both methods when the TOA noise power was less than $35 \mathrm{dBm}^{2}$, but its effect became negligible for larger power of $n_{\mathrm{TOA}, i}$, particularly for the CWLS estimator.

Figures 2, 3, and 4 show the MSREs of the RSS-based, TDOA-based, and AOA-based positioning algorithms, respectively, as well as the corresponding CRLBs, versus power of measurement errors. The disturbances in the RSS and AOA measurements were white Gaussian processes with identical variances as in the TOA measurements. As the units of the $\sigma_{\mathrm{RSS}, i}^{2}$ and $\sigma_{\mathrm{AOA}, i}^{2}$ were $\mathrm{m}^{2 a}$ and $\mathrm{rad}^{2}$, they became $\mathrm{dBm}^{2 a}$ and $\mathrm{dBrad}^{2}$ when represented in $\mathrm{dB}$ scales. While the TDOA measurements were Gaussian with covariance matrix of the form

$$
\mathbf{C}_{\mathbf{n}, \mathrm{TDOA}}=\frac{\sigma_{\mathrm{TDOA}}^{2}}{2}\left[\begin{array}{cccc}
2 & 1 & \cdots & 1 \\
1 & 2 & \cdots & 1 \\
\vdots & \vdots & \ddots & \vdots \\
1 & 1 & \cdots & 2
\end{array}\right]
$$

From the figures, we observe that the performance of all the proposed methods approached the corresponding CRLBs for sufficiently small measurement errors, which verified their optimality at sufficiently high SNRs. Moreover, the superiority of the CWLS approach over the NLS scheme was again demonstrated for larger disturbance environments.

Figure 5 shows the MSREs with TDOA-AOA hybrid measurements, where the disturbances in the same type of measurements had identical power with zero mean, and they were uncorrelated with each other. It can be observed that the variances of the CWLS estimator approached the corresponding CRLB for all cases while the NLS scheme failed to produce optimum performance particularly when the AOA noise power was $-10 \mathrm{dBrad}^{2}$. This illustrated that the CWLS estimator for TDOA-AOA hybrid mobile positioning was optimum for uncorrelated TDOA and AOA measurements and was more robust than the NLS method.

The computational complexity of the CWLS and NLS methods was also compared using the average number of floating point operations (FLOPS) provided by MATLAB, and the results are given in Table 2. It is seen that for AOA measurements, the proposed method required fewer FLOPS than the NLS while it needed more FLOPS for RSS and TOA measurements. For TDOA and TDOA-AOA hybrid measurements, both methods had comparable complexity. It is noteworthy to mention that the computational requirements of the CWLS approach can be significantly reduced if we only solve for the Lagrange multiplier whose value is closest to zero as in the LCLS method [15].

\section{CONCLUSIONS}

This paper considers a unified constrained weighted least squares (CWLS)/weighted least squares (WLS) mobile location approach for time-of-arrival (TOA), received signal strength (RSS), time-difference-of-arrival (TDOA), and angle-of-arrival (AOA) measurements. The basic idea is to reorganize the nonlinear equations obtained from the measurements into linear equations. These linear equations are 


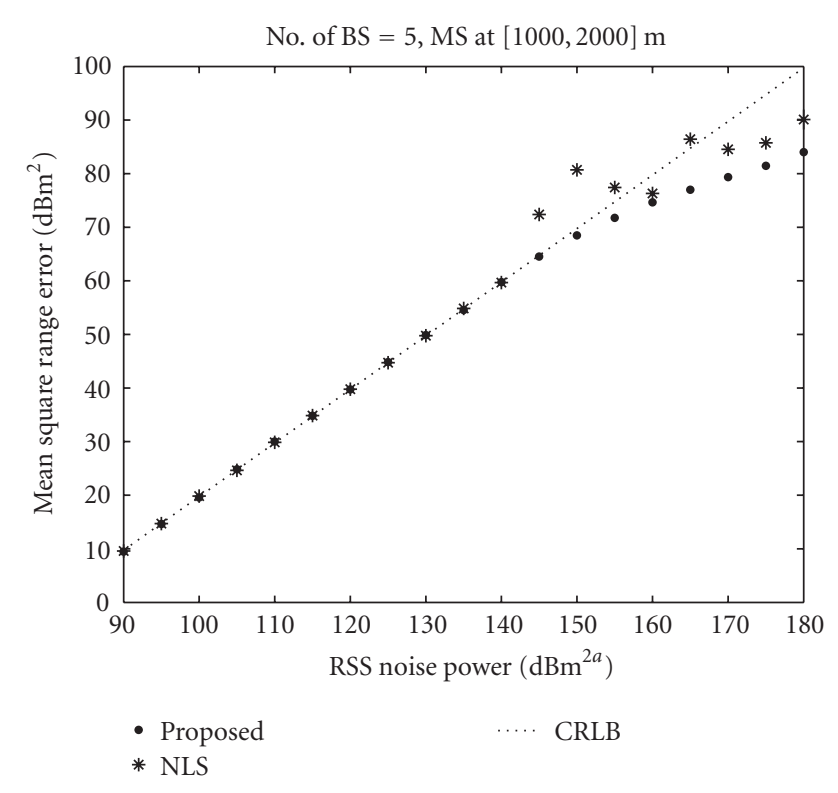

FIGURE 2: Mean square range errors for RSS measurements in uncorrelated noise.

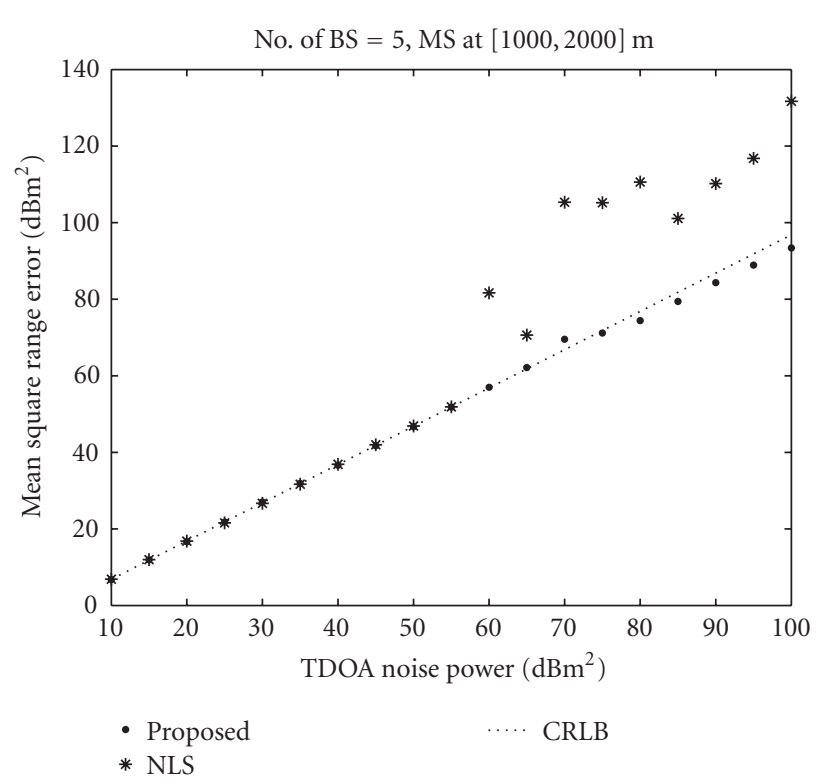

FIgURE 3: Mean square range errors for TDOA measurements in correlated noise.

then solved in an optimum manner with the use of weighted least squares and/or method of Lagrange multipliers. The proposed approach is quite flexible in that it can be easily extended to hybrid measurement cases such as the TDOAAOA. We have proved that for small uncorrelated noise disturbances, the performance of all the proposed CWLS and WLS algorithms attains zero bias and the Cramér-Rao lower

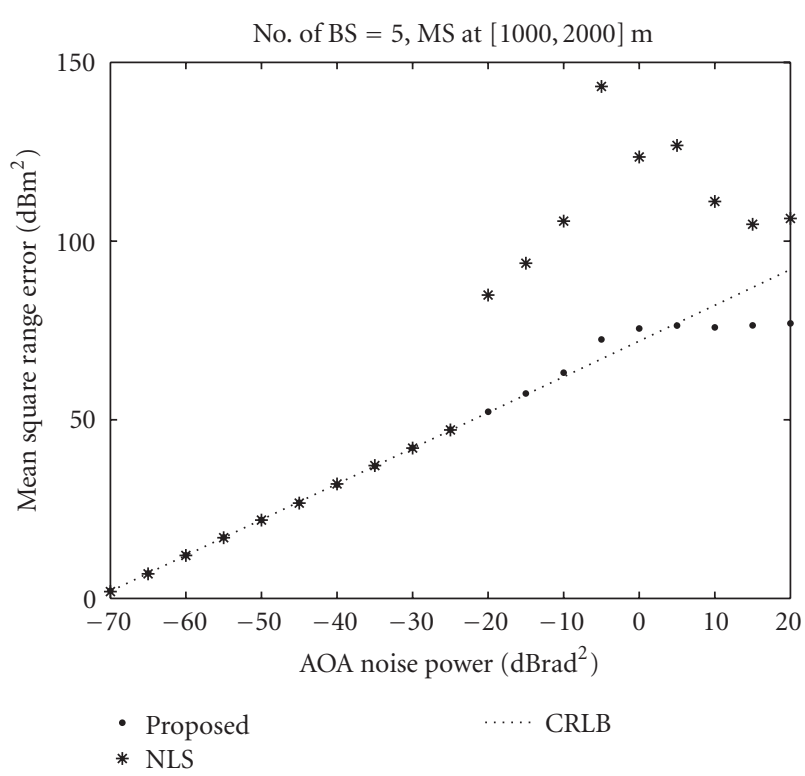

FIgURE 4: Mean square range errors for AOA measurements in uncorrelated noise.

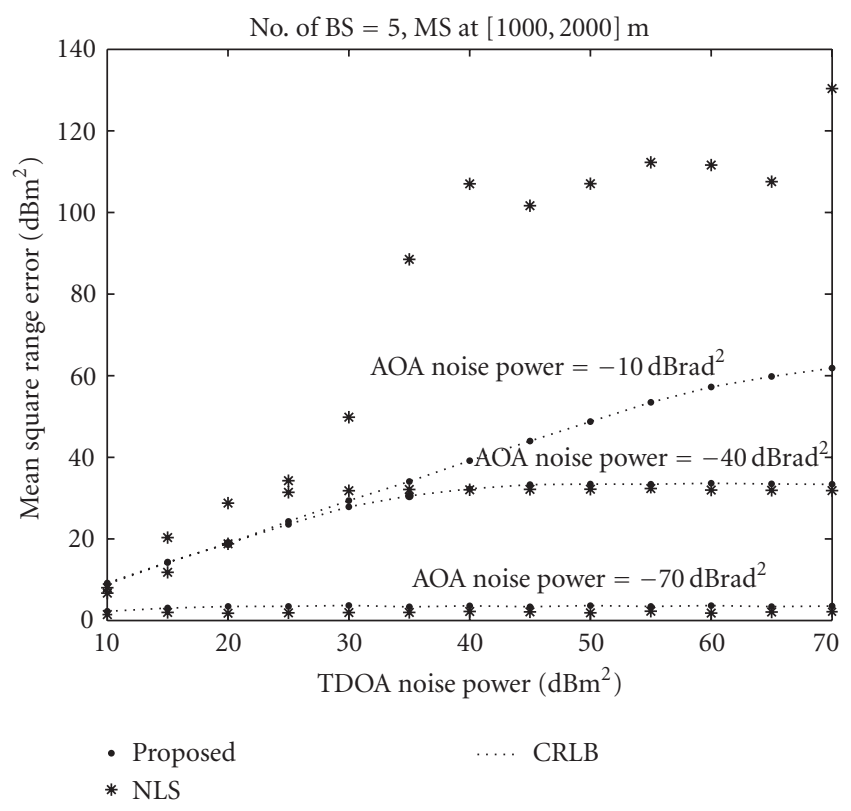

FIGURE 5: Mean square range errors for using both TDOA and AOA measurements.

bound (CRLB) approximately. Simulation results indicate that these theoretical approximation results are accurate, in that the simulated mean square error performance of the developed algorithms closely approaches the CRLBs when the noise variance is small. It is also shown that the proposed approach outperforms the nonlinear least squares scheme in terms of larger optimum operation range. 
TABLE 2: Computational complexity of proposed and NLS methods in terms of FLOPS.

\begin{tabular}{lcc}
\hline & Proposed & NLS \\
\hline TOA & 7125 & 1978 \\
RSS & 6991 & 1393 \\
TDOA & 9892 & 8058 \\
AOA & 1075 & 2667 \\
TDOA-AOA & 11464 & 11994 \\
\hline
\end{tabular}

\section{APPENDICES}

A.

\section{A.1. TDOA}

Following [15], we differentiate (27) and equate the expression to zero:

$$
\frac{\partial \mathcal{L}_{\mathrm{TDOA}}(\breve{\boldsymbol{\vartheta}}, \eta)}{\partial \breve{\boldsymbol{\vartheta}}}=2\left(\mathbf{G}^{T} \mathbf{Y}^{-1} \mathbf{G}+\eta \boldsymbol{\Sigma}\right) \breve{\boldsymbol{\vartheta}}-2 \mathbf{G}^{T} \mathbf{\Upsilon}^{-1} \mathbf{h}=0 .
$$

The solution to (A.1) is

$$
\widehat{\boldsymbol{\vartheta}}=\left(\mathbf{G}^{T} \mathbf{Y}^{-1} \mathbf{G}+\eta \boldsymbol{\Sigma}\right)^{-1} \mathbf{G}^{T} \mathbf{Y}^{-1} \mathbf{h},
$$

where $\eta$ is not yet determined. The Lagrange multiplier is then found by substituting (A.2) into the constraint (23):

$$
\mathbf{h}^{T} \mathbf{Y}^{-1} \mathbf{G}\left(\mathbf{G}^{T} \mathbf{Y}^{-1} \mathbf{G}+\eta \boldsymbol{\Sigma}\right)^{-1} \boldsymbol{\Sigma}\left(\mathbf{G}^{T} \mathbf{Y}^{-1} \mathbf{G}+\eta \boldsymbol{\Sigma}\right)^{-1} \mathbf{G}^{T} \mathbf{Y} \mathbf{h}=0
$$

Using eigenvalue factorization, the matrix $\mathbf{G}^{T} \mathbf{Y}^{-1} \mathbf{G} \boldsymbol{\Sigma}$ can be diagonalized as

$$
\mathbf{G}^{T} \mathbf{Y}^{-1} \mathbf{G} \boldsymbol{\Sigma}=\mathbf{S D S}^{-1}
$$

where $\mathbf{D}=\operatorname{diag}\left(\zeta_{1}, \zeta_{2}, \zeta_{3}\right)$ and $\zeta_{i}, i=1,2,3$, are the eigenvalues of the matrix $\mathbf{G}^{T} \mathbf{Y}^{-1} \mathbf{G} \boldsymbol{\Sigma}$. Substituting (A.4) into (A.3), the constraint can be rewritten as

$$
\boldsymbol{\alpha}^{T}\left(\mathbf{D}+\eta \mathbf{I}_{3}\right)^{-2} \boldsymbol{\beta}=0,
$$

where $\boldsymbol{\alpha}=\mathbf{S}^{T} \boldsymbol{\Sigma} \mathbf{G}^{T} \mathbf{Y}^{-1} \mathbf{h}=\left[\alpha_{1}, \alpha_{2}, \alpha_{3}\right]^{T}$ and $\boldsymbol{\beta}=\mathbf{S}^{-1} \mathbf{G}^{T} \mathbf{Y}^{-1} \mathbf{h}=$ $\left[\beta_{1}, \beta_{2}, \beta_{3}\right]^{T}$. Simplifying (A.5) gives (29).

\section{A.2. RSS}

The minimum of (44) is obtained by differentiating $\mathcal{L}_{\mathrm{RSS}}(\breve{\boldsymbol{\theta}}$, $\lambda$ ) with respect to $\breve{\boldsymbol{\theta}}$ and then equating the resultant expressions to zero:

$$
\frac{\partial \mathcal{L}_{\mathrm{RSS}}(\breve{\boldsymbol{\theta}}, \lambda)}{\partial \boldsymbol{\theta}}=2\left(\mathbf{A}^{T} \boldsymbol{\Psi}^{-1} \mathbf{A}+\lambda \mathbf{P}\right) \breve{\boldsymbol{\theta}}-2 \mathbf{A}^{T} \boldsymbol{\Psi}^{-1} \mathbf{b}+\lambda \mathbf{q}=0 .
$$

The solution to (A.6) is

$$
\widehat{\boldsymbol{\theta}}=\left(\mathbf{A}^{T} \boldsymbol{\Psi}^{-1} \mathbf{A}+\lambda \mathbf{P}\right)^{-1}\left(\mathbf{A}^{T} \Psi^{-1} \mathbf{b}-\frac{\lambda}{2} \mathbf{q}\right),
$$

where $\lambda$ is not determined yet. To find $\lambda$, we substitute (A.7) into the equality constraint of (36):

$$
\begin{aligned}
& \mathbf{q}^{T}\left(\mathbf{A}^{T} \boldsymbol{\Psi}^{-1} \mathbf{A}+\lambda \mathbf{P}\right)^{-1}\left(\mathbf{A}^{T} \boldsymbol{\Psi}^{-1} \mathbf{b}-\frac{\lambda}{2} \mathbf{q}\right)+\left(\mathbf{b}^{T} \boldsymbol{\Psi}^{-1} \mathbf{A}-\frac{\lambda}{2} \mathbf{q}^{T}\right) \\
& \quad \times\left(\mathbf{A}^{T} \boldsymbol{\Psi}^{-1} \mathbf{A}+\lambda \mathbf{P}\right)^{-1} \mathbf{P}\left(\mathbf{A}^{T} \boldsymbol{\Psi}^{-1} \mathbf{A}+\lambda \mathbf{P}\right)^{-1} \\
& \quad \times\left(\mathbf{A}^{T} \boldsymbol{\Psi}^{-1} \mathbf{b}-\frac{\lambda}{2} \mathbf{q}\right)=0
\end{aligned}
$$

Note that the matrix $\left(\mathbf{A}^{T} \Psi^{-1} \mathbf{A}\right)^{-1} \mathbf{P}$ can be diagonalized as

$$
\left(\mathbf{A}^{T} \Psi^{-1} \mathbf{A}\right)^{-1} \mathbf{P}=\mathbf{U} \mathbf{\Lambda} \mathbf{U}^{-1},
$$

where $\boldsymbol{\Lambda}=\operatorname{diag}\left(\gamma_{1}, \gamma_{2}, \gamma_{3}\right)$, and $\gamma_{i}, i=1,2,3$, are the eigenvalues of the matrix $\left(\mathbf{A}^{T} \boldsymbol{\Psi}^{-1} \mathbf{A}\right)^{-1} \mathbf{P}$. Substituting (A.9) into $\left(\mathbf{A}^{T} \Psi^{-1} \mathbf{A}+\lambda \mathbf{P}\right)^{-1}$ gives

$$
\left(\mathbf{A}^{T} \boldsymbol{\Psi}^{-1} \mathbf{A}+\lambda \mathbf{P}\right)^{-1}=\mathbf{U}\left(\mathbf{I}_{3}+\lambda \boldsymbol{\Lambda}\right)^{-1} \mathbf{U}^{-1}\left(\mathbf{A}^{T} \boldsymbol{\Psi}^{-1} \mathbf{A}\right)^{-1}
$$

Putting (A.10) into (A.8), we get

$$
\begin{aligned}
\mathbf{c}^{T}\left(\mathbf{I}_{\mathbf{3}}\right. & +\lambda \boldsymbol{\Lambda})^{-1} \mathbf{f}-\frac{\lambda}{2} \mathbf{c}^{T}\left(\mathbf{I}_{\mathbf{3}}+\lambda \boldsymbol{\Lambda}\right)^{-1} \mathbf{g} \\
& +\mathbf{e}^{T}\left(\mathbf{I}_{\mathbf{3}}+\lambda \boldsymbol{\Lambda}\right)^{-1} \boldsymbol{\Lambda}\left(\mathbf{I}_{\mathbf{3}}+\lambda \boldsymbol{\Lambda}\right)^{-1} \mathbf{f} \\
& -\frac{\lambda}{2} \mathbf{e}^{T}\left(\mathbf{I}_{\mathbf{3}}+\lambda \boldsymbol{\Lambda}\right)^{-1} \boldsymbol{\Lambda}\left(\mathbf{I}_{\mathbf{3}}+\lambda \boldsymbol{\Lambda}\right)^{-1} \mathbf{g} \\
& -\frac{\lambda}{2} \mathbf{c}^{T}\left(\mathbf{I}_{\mathbf{3}}+\lambda \boldsymbol{\Lambda}\right)^{-1} \boldsymbol{\Lambda}\left(\mathbf{I}_{\mathbf{3}}+\lambda \boldsymbol{\Lambda}\right)^{-1} \mathbf{f} \\
& +\frac{\lambda^{2}}{4} \mathbf{c}^{T}\left(\mathbf{I}_{\mathbf{3}}+\lambda \boldsymbol{\Lambda}\right)^{-1} \boldsymbol{\Lambda}\left(\mathbf{I}_{\mathbf{3}}+\lambda \boldsymbol{\Lambda}\right)^{-1} \mathbf{g}=0,
\end{aligned}
$$

where

$$
\begin{aligned}
\mathbf{c}^{T} & =\mathbf{q}^{T} \mathbf{U}=\left[c_{1}, c_{2}, c_{3}\right], \\
\mathbf{g} & =\mathbf{U}^{-1}\left(\mathbf{A}^{T} \boldsymbol{\Psi}^{-1} \mathbf{A}\right)^{-1} \mathbf{q}=\left[g_{1}, g_{2}, g_{3}\right]^{T}, \\
\mathbf{e}^{T} & =\mathbf{b}^{T} \boldsymbol{\Psi}^{-1} \mathbf{A} \mathbf{U}=\left[e_{1}, e_{2}, e_{3}\right], \\
\mathbf{f} & =\mathbf{U}^{-1}\left(\mathbf{A}^{T} \boldsymbol{\Psi}^{-1} \mathbf{A}\right)^{-1} \mathbf{A}^{T} \boldsymbol{\Psi}^{-1} \mathbf{b}=\left[f_{1}, f_{2}, f_{3}\right]^{T} .
\end{aligned}
$$


Since the matrix $\left(\mathbf{A}^{T} \Psi^{-1} \mathbf{A}\right)^{-1} \mathbf{P}$ is of rank 2, one of its eigenvalues, say, $\gamma_{3}$, must be zero. After expanding (A.11) and putting $\gamma_{3}=0$, (A.11) can be simplified to (46).

\section{B.}

For notation convenience, $J_{\mathrm{TDOA}}\left(\breve{\boldsymbol{\vartheta}}_{1}\right), J_{\mathrm{RSS}}(\breve{\mathbf{x}}), J_{\mathrm{AOA}}(\breve{\mathbf{x}})$, and $J_{\mathrm{TDOA}-\mathrm{AOA}}\left(\breve{\boldsymbol{\vartheta}}_{1}\right)$ are written as $J_{\mathrm{TDOA}}, J_{\mathrm{RSS}}, J_{\mathrm{AOA}}$, and $J_{\mathrm{TDOA}-\mathrm{AOA}}$, respectively.

\section{B.1. TDOA}

Differentiate (67) with respect to $\breve{\vartheta}_{1}$ :

$$
\begin{aligned}
\frac{\partial J_{\mathrm{TDOA}}}{\partial \breve{\boldsymbol{\vartheta}}_{1}}= & 2\left[\mathbf{S}^{T}+\left(\breve{\boldsymbol{\vartheta}}_{1}^{T} \breve{\boldsymbol{\vartheta}}_{1}\right)^{-1 / 2} \breve{\boldsymbol{\vartheta}}_{1} \mathbf{r}_{\mathrm{TDOA}}^{T}\right] \\
& \times \mathbf{Y}^{-1}\left[\mathbf{S} \breve{\boldsymbol{\vartheta}}_{1}+\left(\breve{\boldsymbol{\vartheta}}_{1}^{T} \breve{\boldsymbol{\vartheta}}_{1}\right)^{1 / 2} \mathbf{r}_{\mathrm{TDOA}}-\mathbf{h}\right]
\end{aligned}
$$

If the derivative of $J_{\text {TDOA }}$ is located at the true source position $\boldsymbol{\vartheta}_{1}$, assuming that the disturbance to the TDOA measurements is relatively small so that $\left\{n_{\mathrm{TDOA}, i}^{2}\right\}$ can be ignored, then (B.1) becomes

$$
\begin{array}{r}
\left.\frac{\partial J_{\mathrm{TDOA}}}{\partial \breve{\boldsymbol{\vartheta}}_{1}}\right|_{\breve{\vartheta}_{1}=\boldsymbol{\vartheta}_{1}} \approx 2\left[\mathbf{S}^{T}+d_{1}^{-1} \boldsymbol{\vartheta}_{1}\left(\mathbf{s}_{1}-d_{1} \mathbf{1}_{M-1}\right)^{T}\right] \mathbf{\Upsilon}^{-1}\left(\mathbf{s}_{1} \odot \mathbf{n}_{\mathrm{TDOA}}\right) \\
=2\left[\mathbf{S}^{T}+\boldsymbol{\vartheta}_{1}\left(d_{1}^{-1} \mathbf{s}_{1}^{T}-\mathbf{1}_{M-1}^{T}\right)\right] \mathbf{Y}^{-1}\left(\mathbf{s}_{1} \odot \mathbf{n}_{\mathrm{TDOA}}\right)
\end{array}
$$

Taking the expected value on both sides of (B.2) and then applying the fact that $E\left[\mathbf{n}_{\mathrm{TDOA}}\right]=\mathbf{0}_{M-1}$ gives

$$
\begin{aligned}
& \left.E\left[\frac{\partial J_{\mathrm{TDOA}}}{\partial \breve{\boldsymbol{\vartheta}}_{1}}\right]\right|_{\breve{\vartheta}_{1}=\boldsymbol{\vartheta}_{1}} \\
& \quad \approx 2\left[\mathbf{S}^{T}+\boldsymbol{\vartheta}_{1}\left(d_{1}^{-1} \mathbf{s}_{1}^{T}-\mathbf{1}_{M-1}^{T}\right)\right] \mathbf{Y}^{-1}\left(\mathbf{s}_{1} \odot E\left[\mathbf{n}_{\mathrm{TDOA}}\right]\right) \\
& \quad=\mathbf{0}_{2} .
\end{aligned}
$$

Substituting (B.3) into (64) yields

$$
E\left[\hat{\boldsymbol{\vartheta}}_{1}\right] \approx \boldsymbol{\vartheta}_{1}
$$

which indicates that the estimator is unbiased for sufficiently small measurement errors.

Multiplying (B.2) by its transpose and then taking the expected value yields

$$
\begin{aligned}
\left.E\left[\left(\frac{\partial J_{\mathrm{TDOA}}}{\partial \breve{\vartheta}_{1}}\right)\left(\frac{\partial J_{\mathrm{TDOA}}}{\partial \breve{\vartheta}_{1}}\right)^{T}\right]\right|_{\breve{\vartheta}_{1}=\boldsymbol{\vartheta}_{1}} \\
\approx 4\left[\mathbf{S}^{T}+\boldsymbol{\vartheta}_{1}\left(d_{1}^{-1} \mathbf{s}_{1}^{T}-\mathbf{1}_{M-1}^{T}\right)\right] \\
\quad \times \mathbf{\Upsilon}^{-1}\left(\mathbf{s}_{1} \mathbf{s}_{1}^{T} \odot \mathbf{C}_{\mathbf{n}, \mathrm{TDOA}}\right) \mathbf{Y}^{-1}\left[\mathbf{S}+\left(d_{1}^{-1} \mathbf{s}_{1}-\mathbf{1}_{M-1}\right) \boldsymbol{\vartheta}_{1}^{T}\right] \\
=4\left[\mathbf{S}^{T}+\boldsymbol{\vartheta}_{1}\left(d_{1}^{-1} \mathbf{s}_{1}^{T}-\mathbf{1}_{M-1}^{T}\right)\right] \mathbf{Y}^{-1}\left[\mathbf{S}+\left(d_{1}^{-1} \mathbf{s}_{1}-\mathbf{1}_{M-1}\right) \boldsymbol{\vartheta}_{1}^{T}\right]
\end{aligned}
$$

Then differentiating (B.1) with respect to $\breve{x}$, one of the variables in $\breve{\vartheta}_{1}$, by using product rule [29], we get

$$
\begin{aligned}
& \frac{\partial}{\partial \breve{x}}\left(\frac{\partial J_{\mathrm{TDOA}}}{\partial \breve{\mathfrak{\vartheta}}_{1}}\right)=2\left[\mathbf{S}^{T}+\left(\breve{\boldsymbol{\vartheta}}_{1}^{T} \breve{\mathfrak{\vartheta}}_{1}\right)^{-1 / 2} \breve{\mathfrak{\vartheta}}_{1} \mathbf{r}_{\mathrm{TDOA}}^{T}\right] \\
& \times \mathbf{Y}^{-1} \frac{\partial}{\partial \breve{x}}\left[\mathbf{S} \breve{\vartheta}_{1}+\left(\breve{\boldsymbol{\vartheta}}_{1}^{T} \breve{\mathfrak{\vartheta}}_{1}\right)^{1 / 2} \mathbf{r}_{\mathrm{TDOA}}-\mathbf{h}\right] \\
& +2 \frac{\partial}{\partial \breve{x}}\left[\mathbf{S}^{T}+\left(\breve{\boldsymbol{\vartheta}}_{1}^{T} \breve{\vartheta}_{1}\right)^{-1 / 2} \breve{\vartheta}_{1} \mathbf{r}_{\mathrm{TDOA}}^{T}\right] \\
& \times\left\{\mathbf{Y}^{-1}\left[\mathbf{S} \breve{\mathfrak{\vartheta}}_{1}+\left(\breve{\boldsymbol{\vartheta}}_{1}^{T} \breve{\boldsymbol{\vartheta}}_{1}\right)^{1 / 2} \mathbf{r}_{\mathrm{TDOA}}-\mathbf{h}\right]\right\} \\
& =2\left[\mathbf{S}^{T}+\left(\breve{\vartheta}_{1}^{T} \breve{\vartheta}_{1}\right)^{-1 / 2} \breve{\vartheta}_{1} \mathbf{r}_{\mathrm{TDOA}}^{T}\right] \\
& \times \mathbf{Y}^{-1}\left[\mathbf{S}\left[\begin{array}{l}
1 \\
0
\end{array}\right]+\mathbf{r}_{\mathrm{TDOA}}\left(\breve{\mathfrak{\vartheta}}_{1}^{T} \breve{\mathfrak{\vartheta}}_{1}\right)^{-1 / 2}\left(\breve{x}-x_{1}\right)\right] \\
& +2 \frac{\partial}{\partial \breve{x}}\left[\mathbf{S}^{T}+\left(\breve{\mathfrak{\vartheta}}_{1}^{T} \breve{\vartheta}_{1}\right)^{-1 / 2} \breve{\boldsymbol{\vartheta}}_{1} \mathbf{r}_{\mathrm{TDOA}}^{T}\right] \\
& \times\left\{\boldsymbol{Y}^{-1}\left[\mathbf{S} \breve{\vartheta}_{1}+\left(\breve{\boldsymbol{\vartheta}}_{1}^{T} \breve{\vartheta}_{1}\right)^{1 / 2} \mathbf{r}_{\mathrm{TDOA}}-\mathbf{h}\right]\right\} .
\end{aligned}
$$

By substituting the true source location $\boldsymbol{\vartheta}_{1}$ into (B.6) and ignoring the square of the measurement errors $\left\{n_{\mathrm{TDOA}, i}^{2}\right\}$, we obtain

$$
\begin{aligned}
& \left.\frac{\partial}{\partial \breve{x}}\left(\frac{\partial J_{\mathrm{TDOA}}}{\partial \breve{\vartheta}_{1}}\right)\right|_{\breve{\vartheta}_{1}=\boldsymbol{\vartheta}_{1}} \\
& =2\left[\mathbf{S}^{T}+d_{1}^{-1} \boldsymbol{\vartheta}_{1} \mathbf{r}_{\mathrm{TDOA}}^{T}\right] \\
& \times \mathbf{Y}^{-1}\left[\mathbf{S}\left[\begin{array}{l}
1 \\
0
\end{array}\right]+d_{1}^{-1} \mathbf{r}_{\mathrm{TDOA}}\left(x-x_{1}\right)\right] \\
& +\left.2 \frac{\partial}{\partial \breve{x}}\left[\mathbf{S}^{T}+\left(\breve{\boldsymbol{\vartheta}}_{1}^{T} \breve{\boldsymbol{\vartheta}}_{1}\right)^{-1 / 2} \breve{\boldsymbol{\vartheta}}_{1} \mathbf{r}_{\mathrm{TDOA}}^{T}\right]\right|_{\breve{\vartheta}_{1}=\boldsymbol{\vartheta}_{1}} \\
& \times \mathbf{\Upsilon}^{-1}\left(\mathbf{s}_{1} \odot \mathbf{n}_{\mathrm{TDOA}}\right) \\
& \approx 2\left[\mathbf{S}^{T}+d_{1}^{-1} \boldsymbol{\vartheta}_{1}\left(\mathbf{s}_{1}^{T}-d_{1} \mathbf{1}_{M-1}^{T}\right)\right] \\
& \times \mathbf{Y}^{-1}\left[\mathbf{S}\left[\begin{array}{l}
1 \\
0
\end{array}\right]+d_{1}^{-1}\left(\mathbf{s}_{1}-d_{1} \mathbf{1}_{M-1}\right)\left(x-x_{1}\right)\right] \\
& +2\left[\mathbf{S}^{T}+d_{1}^{-1} \boldsymbol{\vartheta}_{1}\left(\mathbf{s}_{1}^{T}-d_{1} \mathbf{1}_{M-1}^{T}\right)\right] d_{1}^{-1} \mathbf{Y}^{-1} \mathbf{n}_{\mathrm{TDOA}}\left(x-x_{1}\right) \\
& +2 d_{1}^{-1} \boldsymbol{\vartheta}_{1} \mathbf{n}_{\mathrm{TDOA}}^{T} \mathbf{\Upsilon}^{-1} \\
& \times\left[\mathbf{S}\left[\begin{array}{l}
1 \\
0
\end{array}\right]+d_{1}^{-1}\left(\mathbf{s}_{1}-d_{1} \mathbf{1}_{M-1}\right)\left(x-x_{1}\right)\right] \\
& +\left.2 \frac{\partial}{\partial \breve{x}}\left[\mathbf{S}^{T}+\left(\breve{\mathfrak{\vartheta}}_{1}^{T} \breve{\vartheta}_{1}\right)^{-1 / 2} \breve{\mathfrak{\vartheta}}_{1} \mathbf{r}_{\mathrm{TDOA}}^{T}\right]\right|_{\breve{\mathfrak{\vartheta}}_{1}=\boldsymbol{\vartheta}_{1}} \\
& \times \mathbf{Y}^{-1}\left(\mathbf{s}_{1} \odot \mathbf{n}_{\mathrm{TDOA}}\right) \text {. }
\end{aligned}
$$


Taking the expected value on both sides of (B.7) and applying the fact that $E\left[\mathbf{n}_{\mathrm{TDOA}}\right]=\mathbf{0}_{M-1}$ gives

$$
\begin{aligned}
\left.E\left[\frac{\partial}{\partial \breve{x}}\left(\frac{\partial J_{\mathrm{TDOA}}}{\partial \breve{\vartheta}_{1}}\right)\right]\right|_{\breve{\mathfrak{\vartheta}}_{1}=\mathbf{\vartheta}_{1}} & \\
\approx & 2\left[\mathbf{S}^{T}+d_{1}^{-1} \mathbf{\vartheta}_{1}\left(\mathbf{s}_{1}^{T}-d_{1} \mathbf{1}_{M-1}^{T}\right)\right] \\
& \times \mathbf{Y}^{-1}\left[\mathbf{S}\left[\begin{array}{l}
1 \\
0
\end{array}\right]+d_{1}^{-1}\left(\mathbf{s}_{1}-d_{1} \mathbf{1}_{M-1}\right)\left(x-x_{1}\right)\right]
\end{aligned}
$$

Similarly, repeating the derivation in (B.6), (B.7), and (B.8) with the variable $\breve{y}$,

$$
\begin{aligned}
\left.E\left[\frac{\partial}{\partial \breve{y}}\left(\frac{\partial J_{\mathrm{TDOA}}}{\partial \breve{\vartheta}_{1}}\right)\right]\right|_{\breve{\vartheta}_{1}=\boldsymbol{\vartheta}_{1}} \\
\approx 2\left[\mathbf{S}^{T}+d_{1}^{-1} \boldsymbol{\vartheta}_{1}\left(\mathbf{s}_{1}^{T}-d_{1} \mathbf{1}_{M-1}^{T}\right)\right] \\
\quad \times \mathbf{\Upsilon}^{-1}\left[\mathbf{S}\left[\begin{array}{l}
0 \\
1
\end{array}\right]+d_{1}^{-1}\left(\mathbf{s}_{1}-d_{1} \mathbf{1}_{M-1}\right)\left(y-y_{1}\right)\right]
\end{aligned}
$$

We also get

$$
\begin{aligned}
& \left.E\left[\frac{\partial^{2} J_{\mathrm{TDOA}}}{\partial \breve{\mathfrak{\vartheta}}_{1} \partial \breve{\boldsymbol{\vartheta}}_{1}^{T}}\right]\right|_{\breve{\mathfrak{\vartheta}}_{1}=\boldsymbol{\vartheta}_{1}} \\
& \quad=\left[\left.\left.E\left[\frac{\partial}{\partial \breve{x}}\left(\frac{\partial J_{\mathrm{TDOA}}}{\partial \breve{\boldsymbol{\vartheta}}_{1}}\right)\right]\right|_{\breve{\mathfrak{\vartheta}}_{1}=\boldsymbol{\vartheta}_{1}} E\left[\frac{\partial}{\partial \breve{y}}\left(\frac{\partial J_{\mathrm{TDOA}}}{\partial \breve{\boldsymbol{\vartheta}}_{1}}\right)\right]\right|_{\breve{\mathfrak{\vartheta}}_{1}=\boldsymbol{\vartheta}_{1}}\right] .
\end{aligned}
$$

Hence substituting (B.8) and (B.9) into (B.10) yields

$$
\begin{aligned}
\left.E\left[\frac{\partial^{2} J_{\mathrm{TDOA}}}{\partial \breve{\boldsymbol{\vartheta}}_{1} \partial \breve{\vartheta}_{1}^{T}}\right]\right|_{\breve{\vartheta}_{1}=\boldsymbol{\vartheta}_{1}} \approx & 2\left[\mathbf{S}^{T}+d_{1}^{-1} \boldsymbol{\vartheta}_{1}\left(\mathbf{s}_{1}^{T}-d_{1} \mathbf{1}_{M-1}^{T}\right)\right] \\
& \times \mathbf{\Upsilon}^{-1}\left[\mathbf{S}+d_{1}^{-1}\left(\mathbf{s}_{1}-d_{1} \mathbf{1}_{M-1}\right) \boldsymbol{\vartheta}_{1}^{T}\right]
\end{aligned}
$$

Then by substituting (B.5) and (B.11) into (65), the covariance matrix for the MS position estimate $\boldsymbol{\vartheta}_{1}$ is obtained as

$$
\begin{aligned}
\mathbf{C}_{\boldsymbol{\vartheta}_{1}} \approx & \left\{\left[\mathbf{S}^{T}+d_{1}^{-1} \boldsymbol{\vartheta}_{1}\left(\mathbf{s}_{1}^{T}-d_{1} \mathbf{1}_{M-1}^{T}\right)\right]\right. \\
& \left.\times \mathbf{Y}^{-1}\left[\mathbf{S}+d_{1}^{-1}\left(\mathbf{s}_{1}-d_{1} \mathbf{1}_{M-1}\right) \boldsymbol{\vartheta}_{1}^{T}\right]\right\}^{-1}
\end{aligned}
$$

Substituting $\mathbf{x}-\mathbf{x}_{1}$ back to $\boldsymbol{\vartheta}_{1}$ in (B.4) and (B.12) and applying the fact that $\mathbf{C}_{\mathbf{x}}=\mathbf{C}_{\boldsymbol{\vartheta}_{1}}$ gives (69) and (70).

\section{B.2. RSS}

Differentiate (77) with respect to $\breve{\mathbf{x}}$,

$$
\frac{\partial J_{\mathrm{RSS}}}{\partial \breve{\mathbf{x}}}=2\left(\mathbf{X}_{\mathrm{BS}}^{T}-\breve{\mathbf{x}} \mathbf{1}_{M}^{T}\right) \boldsymbol{\Psi}^{-1}\left[\mathbf{X}_{\mathrm{BS}} \breve{\mathbf{x}}-0.5\left(\breve{\mathbf{x}}^{T} \breve{\mathbf{x}}\right) \mathbf{1}_{M}-\mathbf{b}\right] .
$$

Assuming that the disturbances due to the RSS measurements are sufficiently small such that $\left\{n_{\mathrm{RSS}, i}^{2}\right\}$ can be ignored, the derivative of $J_{\text {RSS }}$ evaluated at the true MS position $\mathbf{x}$ becomes

$$
\left.\frac{\partial J_{\mathrm{RSS}}}{\partial \breve{\mathbf{x}}}\right|_{\breve{\mathbf{x}}=\mathbf{x}} \approx 2\left(\mathbf{X}_{\mathrm{BS}}^{T}-\mathbf{x} \mathbf{1}_{M}^{T}\right) \mathbf{\Psi}^{-1}\left(\mathbf{s}_{2} \odot \mathbf{n}_{\mathrm{RSS}}\right)
$$

Take the expected value on both sides of (B.14) and then apply the fact that $E\left[\mathbf{n}_{\mathrm{RSS}}\right]=\mathbf{0}_{M}$, we get

$$
\left.E\left[\frac{\partial J_{\mathrm{RSS}}}{\partial \breve{\mathbf{x}}}\right]\right|_{\breve{\mathbf{x}}=\mathbf{x}} \approx 2\left(\mathbf{X}_{\mathrm{BS}}^{T}-\mathbf{x} \mathbf{1}_{M}^{T}\right) \boldsymbol{\Psi}^{-1}\left(\mathbf{s}_{2} \odot E\left[\mathbf{n}_{\mathrm{RSS}}\right]\right)=\mathbf{0}_{2}
$$

Substituting (B.15) into (64) yields (79).

Multiplying (B.14) by its transpose and then taking the expected value yields

$$
\begin{gathered}
\left.E\left[\left(\frac{\partial J_{\mathrm{RSS}}}{\partial \breve{\mathbf{x}}}\right)\left(\frac{\partial J_{\mathrm{RSS}}}{\partial \breve{\mathbf{x}}}\right)^{T}\right]\right|_{\breve{\mathbf{x}}=\mathbf{x}} \\
\quad \approx 4\left(\mathbf{X}_{\mathrm{BS}}^{T}-\breve{\mathbf{x}} \mathbf{1}_{M}^{T}\right) \boldsymbol{\Psi}^{-1}\left(\mathbf{s}_{2} \mathbf{s}_{2}^{T} \odot \mathbf{C}_{\mathbf{n}, \mathrm{RSS}}\right) \boldsymbol{\Psi}^{-1}\left(\mathbf{X}_{\mathrm{BS}}-\mathbf{1}_{M} \breve{\mathbf{x}}^{T}\right) \\
\quad=4\left(\mathbf{X}_{\mathrm{BS}}^{T}-\breve{\mathbf{x}} \mathbf{1}_{M}^{T}\right) \Psi^{-1}\left(\mathbf{X}_{\mathrm{BS}}-\mathbf{1}_{M} \breve{\mathbf{x}}^{T}\right) .
\end{gathered}
$$

On the other hand, differentiating (B.13) with respect to $\breve{x}$, the first variable in $\breve{\mathbf{x}}$, and with the use of product rule [29], we get

$$
\begin{aligned}
\frac{\partial}{\partial \breve{x}}\left(\frac{\partial J_{\mathrm{RSS}}}{\partial \breve{\mathbf{x}}}\right) & \\
= & 2\left(\mathbf{X}_{\mathrm{BS}}^{T}-\breve{\mathbf{x}} \mathbf{1}_{M}^{T}\right) \boldsymbol{\Psi}^{-1} \frac{\partial}{\partial \breve{x}}\left[\mathbf{X}_{\mathrm{BS}} \breve{\mathbf{x}}-0.5\left(\breve{\mathbf{x}}^{T} \breve{\mathbf{x}}\right) \mathbf{1}_{M}-\mathbf{b}\right] \\
& +2\left[\frac{\partial}{\partial \breve{x}}\left(\mathbf{X}_{\mathrm{BS}}^{T}-\breve{\mathbf{x}}_{M}^{T}\right)\right] \boldsymbol{\Psi}^{-1}\left[\mathbf{X}_{\mathrm{BS}} \breve{\mathbf{x}}-0.5\left(\breve{\mathbf{x}}^{T} \breve{\mathbf{x}}\right) \mathbf{1}_{M}-\mathbf{b}\right] \\
= & 2\left(\mathbf{X}_{\mathrm{BS}}^{T}-\breve{\mathbf{x}} \mathbf{1}_{M}^{T}\right) \boldsymbol{\Psi}^{-1}\left[\mathbf{X}_{\mathrm{BS}}\left[\begin{array}{l}
1 \\
0
\end{array}\right]-\mathbf{1}_{M} \breve{x}\right] \\
& +2\left[\frac{\partial}{\partial \breve{x}}\left(\mathbf{X}_{\mathrm{BS}}^{T}-\breve{\mathbf{x}} \mathbf{1}_{M}^{T}\right)\right] \boldsymbol{\Psi}^{-1}\left[\mathbf{X}_{\mathrm{BS}} \breve{\mathbf{x}}-0.5\left(\breve{\mathbf{x}}^{T} \breve{\mathbf{x}}\right) \mathbf{1}_{M}-\mathbf{b}\right] .
\end{aligned}
$$


Ignoring the terms of $\left\{n_{\mathrm{RSS}, i}^{2}\right\}$ again, the value of (B.17) computed at $\mathbf{x}$ is

$$
\begin{aligned}
\left.\frac{\partial}{\partial \breve{x}}\left(\frac{\partial J_{\mathrm{RSS}}}{\partial \breve{\mathbf{x}}}\right)\right|_{\breve{\mathbf{x}}=\mathbf{x}}= & 2\left(\mathbf{X}_{\mathrm{BS}}^{T}-\mathbf{x} \mathbf{1}_{M}^{T}\right) \boldsymbol{\Psi}^{-1}\left[\mathbf{X}_{\mathrm{BS}}\left[\begin{array}{l}
1 \\
0
\end{array}\right]-\mathbf{1}_{M} x\right] \\
& +\left.2\left[\frac{\partial}{\partial \breve{x}}\left(\mathbf{X}_{\mathrm{BS}}^{T}-\breve{\mathbf{x}} \mathbf{1}_{M}^{T}\right)\right]\right|_{\breve{\mathbf{x}}=\mathbf{x}} \Psi^{-1}\left(\mathbf{s}_{2} \odot \mathbf{n}_{\mathrm{RSS}}\right)
\end{aligned}
$$

Taking the expected value on both sides of (B.18) and applying $E\left[\mathbf{n}_{\mathrm{RSS}}\right]=\mathbf{0}_{M}$ gives

$\left.E\left[\frac{\partial}{\partial \breve{x}}\left(\frac{\partial J_{\mathrm{RSS}}}{\partial \breve{\mathbf{x}}}\right)\right]\right|_{\breve{\mathbf{x}}=\mathbf{x}} \approx 2\left(\mathbf{X}_{\mathrm{BS}}^{T}-\mathbf{x} \mathbf{1}_{M}^{T}\right) \boldsymbol{\Psi}^{-1}\left[\mathbf{X}_{\mathrm{BS}}\left[\begin{array}{l}1 \\ 0\end{array}\right]-\mathbf{1}_{M} x\right]$

Similarly, repeating the derivations in (B.17)-(B.19) with the second variable $\breve{y}$, we obtain

$\left.E\left[\frac{\partial}{\partial \breve{y}}\left(\frac{\partial J_{\mathrm{RSS}}}{\partial \breve{\mathbf{x}}}\right)\right]\right|_{\breve{\mathbf{x}}=\mathbf{x}} \approx 2\left(\mathbf{X}_{\mathrm{BS}}^{T}-\mathbf{x} \mathbf{1}_{M}^{T}\right) \boldsymbol{\Psi}^{-1}\left[\mathbf{X}_{\mathrm{BS}}\left[\begin{array}{l}0 \\ 1\end{array}\right]-\mathbf{1}_{M} y\right]$

We also have

$$
\begin{aligned}
& \left.E\left[\frac{\partial^{2} J_{\mathrm{RSS}}}{\partial \breve{\mathbf{x}} \partial \breve{\mathbf{x}}^{T}}\right]\right|_{\breve{\mathbf{x}}=\mathbf{x}} \\
& \quad=\left[\left.\left.E\left[\frac{\partial}{\partial \breve{x}}\left(\frac{\partial J_{\mathrm{RSS}}}{\partial \breve{\mathbf{x}}}\right)\right]\right|_{\breve{\mathbf{x}}=\mathbf{x}} E\left[\frac{\partial}{\partial \breve{y}}\left(\frac{\partial J_{\mathrm{RSS}}}{\partial \breve{\mathbf{x}}}\right)\right]\right|_{\breve{\mathbf{x}}=\mathbf{x}}\right] .
\end{aligned}
$$

Substituting (B.19) and (B.20) into (B.21) yields

$$
\left.E\left[\frac{\partial^{2} J_{\mathrm{RSS}}}{\partial \breve{\mathbf{x}} \partial \breve{\mathbf{x}}^{T}}\right]\right|_{\breve{\mathbf{x}}=\mathbf{x}} \approx 2\left(\mathbf{X}_{\mathrm{BS}}^{T}-\mathbf{x} \mathbf{1}_{M}^{T}\right) \boldsymbol{\Psi}^{-1}\left(\mathbf{X}_{\mathrm{BS}}-\mathbf{1}_{M} \mathbf{x}^{T}\right)
$$

Then substituting (B.16) and (B.22) into (65) gives (80).

\section{B.3. $A O A$}

Differentiating (86) with respect to $\breve{\mathbf{x}}$, we get

$$
\frac{\partial J_{\mathrm{AOA}}}{\partial \breve{\mathbf{x}}}=2 \mathbf{H}^{T} \mathbf{\Omega}^{-1}(\mathbf{H} \breve{\mathbf{x}}-\mathbf{k})
$$

Assuming that the disturbances due to the AOA measurements are sufficiently small such that $\left\{n_{\mathrm{AOA}, i}^{2}\right\}$ can be ignored, the derivative of $J_{\mathrm{AOA}}$ evaluated at the true value of $\mathbf{x}$ becomes

$$
\left.\frac{\partial J_{\mathrm{AOA}}}{\partial \breve{\mathbf{x}}}\right|_{\breve{\mathbf{x}}=\mathbf{x}} \approx 2 \mathbf{H}^{T} \boldsymbol{\Omega}^{-1}\left(\mathbf{s}_{3} \odot \mathbf{n}_{\mathrm{AOA}}\right)
$$

Taking the expected value on both sides of (B.24) and then applying the fact that $E\left[\mathbf{n}_{\mathrm{AOA}}\right]=\mathbf{0}_{M}$, we obtain

$$
\left.E\left[\frac{\partial J_{\mathrm{AOA}}}{\partial \breve{\mathbf{x}}}\right]\right|_{\breve{\mathbf{x}}=\mathbf{x}} \approx 2 \mathbf{H}^{T} \boldsymbol{\Omega}^{-1}\left(\mathbf{s}_{3} \odot E\left[\mathbf{n}_{\mathrm{AOA}}\right]\right)=\mathbf{0}_{2}
$$

Substituting (B.25) into (64) gives (87).

Multiplying (B.24) by its transpose and then taking the expected value yields

$$
\begin{aligned}
\left.E\left[\left(\frac{\partial J_{\mathrm{AOA}}}{\partial \breve{\mathbf{x}}}\right)\left(\frac{\partial J_{\mathrm{AOA}}}{\partial \breve{\mathbf{x}}}\right)^{T}\right]\right|_{\breve{\mathbf{x}}=\mathbf{x}} \approx 4 \mathbf{H}^{T} \boldsymbol{\Omega}^{-1}\left(\mathbf{s}_{3} \mathbf{s}_{3}^{T} \odot \mathbf{C}_{\mathbf{n}, \mathrm{AOA}}\right) \boldsymbol{\Omega}^{-1} \mathbf{H} \\
=4 \mathbf{H}^{T} \boldsymbol{\Omega}^{-1} \mathbf{H} .
\end{aligned}
$$

Differentiating (B.23) with respect to $\breve{\mathbf{x}}$, we get

$$
\frac{\partial^{2} J_{\mathrm{AOA}}}{\partial \breve{\mathbf{x}} \partial \breve{\mathbf{x}}^{T}}=2 \mathbf{H}^{T} \mathbf{\Omega}^{-1} \mathbf{H}
$$

Since (B.27) does not contain $\mathbf{x}$ and $\mathbf{n}_{\mathrm{AOA}}$, taking the expected value on both sides of (B.27) yields

$$
\left.E\left[\frac{\partial^{2} J_{\mathrm{AOA}}}{\partial \breve{\mathbf{x}} \partial \breve{\mathbf{x}}^{T}}\right]\right|_{\breve{\mathbf{x}}=\mathbf{x}}=2 \mathbf{H}^{T} \boldsymbol{\Omega}^{-1} \mathbf{H}
$$

Substituting (B.26) and (B.28) into (65) gives (88).

\section{B.4. TDOA-AOA hybrid}

Differentiate (94) with respect to $\breve{\vartheta}_{1}$

$$
\begin{aligned}
& \frac{\partial J_{\mathrm{TDOA}-\mathrm{AOA}}}{\partial \breve{\boldsymbol{\vartheta}}_{1}} \\
& \quad=2\left[\left[\begin{array}{ll}
\mathbf{S}^{T} & \mathbf{H}^{T}
\end{array}\right]+\left(\breve{\boldsymbol{\vartheta}}_{1}^{T} \breve{\mathfrak{\vartheta}}_{1}\right)^{-1 / 2} \breve{\mathfrak{\vartheta}}_{1}\left[\begin{array}{ll}
\mathbf{r}_{\mathrm{TDOA}}^{T} & \mathbf{0}_{M}^{T}
\end{array}\right]\right] \\
& \quad \times \mathbf{W}^{-1}\left[\left[\begin{array}{c}
\mathbf{S} \\
\mathbf{H}
\end{array}\right] \breve{\boldsymbol{\vartheta}}_{1}+\left(\breve{\boldsymbol{\vartheta}}_{1}^{T} \breve{\boldsymbol{\vartheta}}_{1}\right)^{1 / 2}\left[\begin{array}{c}
\mathbf{r}_{\mathrm{TDOA}} \\
\mathbf{0}_{M}
\end{array}\right]-\mathbf{w}\right] .
\end{aligned}
$$


If the derivative of $J_{\text {TDOA-AOA }}$ is located at the true source position $\boldsymbol{\vartheta}_{1}$, assuming that the disturbances are relatively small so that $\left\{n_{\mathrm{TDOA}, i}^{2}\right\}$ and $\left\{n_{\mathrm{AOA}, i}^{2}\right\}$ can be ignored, then (B.29) becomes

$$
\begin{aligned}
& \left.\frac{\partial J_{\mathrm{TDOA}-\mathrm{AOA}}}{\partial \breve{\boldsymbol{\vartheta}}_{1}}\right|_{\breve{\mathfrak{\vartheta}}_{1}=\boldsymbol{\vartheta}_{1}} \\
& \approx 2\left[\left[\begin{array}{ll}
\mathbf{S}^{T} & \mathbf{H}^{T}
\end{array}\right]+d_{1}^{-1} \boldsymbol{\vartheta}_{1}\left(\left[\begin{array}{c}
\mathbf{s}_{1} \\
\mathbf{0}_{M}
\end{array}\right]-d_{1}\left[\begin{array}{c}
\mathbf{1}_{M-1} \\
\mathbf{0}_{M}
\end{array}\right]\right)^{T}\right] \\
& \times \mathbf{W}^{-1}\left(\mathbf{s}_{4} \odot \mathbf{n}_{\text {TDOA-AOA }}\right) \\
& =2\left[\left[\begin{array}{ll}
\mathbf{S}^{T} & \mathbf{H}^{T}
\end{array}\right]+\boldsymbol{\vartheta}_{1}\left(d_{1}^{-1}\left[\begin{array}{c}
\mathbf{s}_{1} \\
\mathbf{0}_{M}
\end{array}\right]-\left[\begin{array}{c}
\mathbf{1}_{M-1} \\
\mathbf{0}_{M}
\end{array}\right]\right)^{T}\right] \\
& \times \mathbf{W}^{-1}\left(\mathbf{s}_{4} \odot \mathbf{n}_{\mathrm{TDOA}-\mathrm{AOA}}\right) \text {. }
\end{aligned}
$$

Taking the expected value on both sides of (B.30) and then applying the fact that $E\left[\mathbf{n}_{\mathrm{TDOA}}\right]=\mathbf{0}_{M-1}$ and $E\left[\mathbf{n}_{\mathrm{AOA}}\right]=\mathbf{0}_{M}$ gives

$$
\begin{aligned}
\left.E\left[\frac{\partial J_{\mathrm{TDOA}-\mathrm{AOA}}}{\partial \breve{\boldsymbol{\vartheta}}_{1}}\right]\right|_{\breve{\vartheta}_{1}=\boldsymbol{\vartheta}_{1}} \\
=2\left[\left[\begin{array}{ll}
\mathbf{S}^{T} & \mathbf{H}^{T}
\end{array}\right]+\boldsymbol{\vartheta}_{1}\left(d_{1}^{-1}\left[\begin{array}{c}
\mathbf{s}_{1} \\
\mathbf{0}_{M}
\end{array}\right]-\left[\begin{array}{c}
\mathbf{1}_{M-1} \\
\mathbf{0}_{M}
\end{array}\right]\right)^{T}\right] \\
\quad \times \mathbf{W}^{-1}\left(\mathbf{s}_{4} \odot \mathbf{n}_{\mathrm{TDOA}-\mathrm{AOA}}\right)=\mathbf{0}_{2}
\end{aligned}
$$

which results in (96) and indicates that the estimator is unbiased for sufficiently small measurement errors.

Multiplying (B.29) by its transpose and then taking the expected value yields

$$
\begin{aligned}
& \left.E\left[\left(\frac{\partial J_{\mathrm{TDOA}-\mathrm{AOA}}}{\partial \breve{\boldsymbol{\vartheta}}_{1}}\right)\left(\frac{\partial J_{\mathrm{TDOA}-\mathrm{AOA}}}{\partial \breve{\boldsymbol{\vartheta}}_{1}}\right)^{T}\right]\right|_{\breve{\mathfrak{\vartheta}}_{1}=\boldsymbol{\vartheta}_{1}} \\
& \approx 4\left[\left[\begin{array}{ll}
\mathbf{S}^{T} & \mathbf{H}^{T}
\end{array}\right]+\boldsymbol{\vartheta}_{1}\left(d_{1}^{-1}\left[\begin{array}{c}
\mathbf{s}_{1} \\
\mathbf{0}_{M}
\end{array}\right]-\left[\begin{array}{c}
\mathbf{1}_{M-1} \\
\mathbf{0}_{M}
\end{array}\right]\right)^{T}\right] \\
& \times \mathbf{W}^{-1}\left(\mathbf{s}_{4} \mathbf{s}_{4}^{T} \odot \mathbf{C}_{\mathbf{n}, \text { TDOA-AOA }}\right) \\
& \times \mathbf{W}^{-1}\left[\left[\begin{array}{c}
\mathbf{S} \\
\mathbf{H}
\end{array}\right]+\left(d_{1}^{-1}\left[\begin{array}{c}
\mathbf{s}_{1} \\
\mathbf{0}_{M}
\end{array}\right]-\left[\begin{array}{c}
\mathbf{1}_{M-1} \\
\mathbf{0}_{M}
\end{array}\right]\right) \boldsymbol{\vartheta}_{1}^{T}\right] \\
& =4\left[\left[\begin{array}{ll}
\mathbf{S}^{T} & \mathbf{H}^{T}
\end{array}\right]+\boldsymbol{\vartheta}_{1}\left(d_{1}^{-1}\left[\begin{array}{c}
\mathbf{s}_{1} \\
\mathbf{0}_{M}
\end{array}\right]-\left[\begin{array}{c}
\mathbf{1}_{M-1} \\
\mathbf{0}_{M}
\end{array}\right]\right)^{T}\right] \\
& \times \mathbf{W}^{-1}\left[\left[\begin{array}{c}
\mathbf{S} \\
\mathbf{H}
\end{array}\right]+\left(d_{1}^{-1}\left[\begin{array}{c}
\mathbf{s}_{1} \\
\mathbf{0}_{M}
\end{array}\right]-\left[\begin{array}{c}
\mathbf{1}_{M-1} \\
\mathbf{0}_{M}
\end{array}\right]\right) \mathbf{\vartheta}_{1}^{T}\right] .
\end{aligned}
$$

Then differentiating (B.29) with respect to $\breve{x}$, one of the variables in $\breve{\vartheta}_{1}$, by using product rule [29], we get

$$
\begin{aligned}
& \frac{\partial}{\partial \breve{x}}\left(\frac{\partial J_{\mathrm{TDOA}-\mathrm{AOA}}}{\partial \breve{\vartheta}_{1}}\right) \\
& =2\left[\left[\begin{array}{ll}
\mathbf{S}^{T} & \mathbf{H}^{T}
\end{array}\right]+\left(\breve{\boldsymbol{\vartheta}}_{1}^{T} \breve{\boldsymbol{\vartheta}}_{1}\right)^{-1 / 2} \breve{\vartheta}_{1}\left[\begin{array}{ll}
\mathbf{r}_{\mathrm{TDOA}}^{T} & \mathbf{0}_{M}^{T}
\end{array}\right]\right] \\
& \times \mathbf{W}^{-1} \frac{\partial}{\partial \breve{x}}\left[\left[\begin{array}{c}
\mathbf{S} \\
\mathbf{H}
\end{array}\right] \breve{\boldsymbol{\vartheta}}_{1}+\left(\breve{\mathfrak{\vartheta}}_{1}^{T} \breve{\mathfrak{\vartheta}}_{1}\right)^{1 / 2}\left[\begin{array}{c}
\mathbf{r}_{\mathrm{TDOA}} \\
\mathbf{0}_{M}
\end{array}\right]-\mathbf{w}\right] \\
& +2 \frac{\partial}{\partial \breve{x}}\left[\left[\begin{array}{ll}
\mathbf{S}^{T} & \mathbf{H}^{T}
\end{array}\right]+\left(\breve{\boldsymbol{\vartheta}}_{1}^{T} \breve{\boldsymbol{\vartheta}}_{1}\right)^{-1 / 2} \breve{\boldsymbol{\vartheta}}_{1}\left[\begin{array}{ll}
\mathbf{r}_{\mathrm{TDOA}}^{T} & \mathbf{0}_{M}^{T}
\end{array}\right]\right] \\
& \times\left\{\mathbf{W}^{-1}\left[\left[\begin{array}{c}
\mathbf{S} \\
\mathbf{H}
\end{array}\right] \breve{\boldsymbol{\vartheta}}_{1}+\left(\breve{\boldsymbol{\vartheta}}_{1}^{T} \breve{\boldsymbol{\vartheta}}_{1}\right)^{1 / 2}\left[\begin{array}{c}
\mathbf{r}_{\mathrm{TDOA}} \\
\mathbf{0}_{M}
\end{array}\right]-\mathbf{w}\right]\right\} \\
& =2\left[\left[\begin{array}{ll}
\mathbf{S}^{T} & \mathbf{H}^{T}
\end{array}\right]+\left(\breve{\boldsymbol{\vartheta}}_{1}^{T} \breve{\boldsymbol{\vartheta}}_{1}\right)^{-1 / 2} \breve{\boldsymbol{\vartheta}}_{1}\left[\begin{array}{ll}
\mathbf{r}_{\mathrm{TDOA}}^{T} & \mathbf{0}_{M}^{T}
\end{array}\right]\right] \\
& \times \mathbf{W}^{-1}\left[\left[\begin{array}{c}
\mathbf{S} \\
\mathbf{H}
\end{array}\right]\left[\begin{array}{l}
1 \\
0
\end{array}\right]+\left[\begin{array}{c}
\mathbf{r}_{\mathrm{TDOA}} \\
\mathbf{0}_{M}^{T}
\end{array}\right]\left(\breve{\boldsymbol{\vartheta}}_{1}^{T} \breve{\boldsymbol{\vartheta}}_{1}\right)^{-1 / 2}\left(\breve{x}-x_{1}\right)\right] \\
& +2 \frac{\partial}{\partial \breve{x}}\left[\left[\begin{array}{ll}
\mathbf{S}^{T} & \mathbf{H}^{T}
\end{array}\right]+\left(\breve{\boldsymbol{\vartheta}}_{1}^{T} \breve{\vartheta}_{1}\right)^{-1 / 2} \breve{\boldsymbol{\vartheta}}_{1}\left[\begin{array}{ll}
\mathbf{r}_{\mathrm{TDOA}}^{T} & \mathbf{0}_{M}^{T}
\end{array}\right]\right] \\
& \times\left\{\mathbf{W}^{-1}\left[\left[\begin{array}{c}
\mathbf{S} \\
\mathbf{H}
\end{array}\right] \breve{\boldsymbol{\vartheta}}_{1}+\left(\breve{\boldsymbol{\vartheta}}_{1}^{T} \breve{\boldsymbol{\vartheta}}_{1}\right)^{1 / 2}\left[\begin{array}{c}
\mathbf{r}_{\mathrm{TDOA}} \\
\mathbf{0}_{M}
\end{array}\right]-\mathbf{w}\right]\right\} .
\end{aligned}
$$

By substituting the true source location $\boldsymbol{\vartheta}_{1}$ into (B.33) and ignoring the square of the measurement errors $\left\{n_{\mathrm{TDOA}, i}^{2}\right\}$ and $\left\{n_{\mathrm{AOA}, i}^{2}\right\}$, we obtain

$$
\begin{aligned}
&\left.\frac{\partial}{\partial \breve{x}}\left(\frac{\partial J_{\mathrm{TDOA}-\mathrm{AOA}}}{\partial \breve{\vartheta}_{1}}\right)\right|_{\breve{\vartheta}_{1}=\mathbf{\vartheta}_{1}} \\
&=2\left[\left[\begin{array}{ll}
\mathbf{S}^{T} & \mathbf{H}^{T}
\end{array}\right]+d_{1}^{-1} \boldsymbol{\vartheta}_{1}\left[\begin{array}{ll}
\mathbf{r}_{\mathrm{TDOA}}^{T} & \mathbf{0}_{M}^{T}
\end{array}\right]\right] \\
& \quad \times \mathbf{W}^{-1}\left[\left[\begin{array}{l}
\mathbf{S} \\
\mathbf{H}
\end{array}\right]\left[\begin{array}{l}
1 \\
0
\end{array}\right]+d_{1}^{-1}\left[\begin{array}{c}
\mathbf{r}_{\mathrm{TDOA}} \\
\mathbf{0}_{M}
\end{array}\right]\left(x-x_{1}\right)\right] \\
& \quad+\left.2 \frac{\partial}{\partial \breve{x}}\left[\left[\begin{array}{ll}
\mathbf{S}^{T} & \mathbf{H}^{T}
\end{array}\right]+\left(\breve{\boldsymbol{\vartheta}}_{1}^{T} \breve{\boldsymbol{\vartheta}}_{1}\right)^{-1 / 2} \breve{\boldsymbol{\vartheta}}_{1}\left[\begin{array}{ll}
\mathbf{r}_{\mathrm{TDOA}}^{T} & \mathbf{0}_{M}^{T}
\end{array}\right]\right]\right|_{\breve{\vartheta}_{1}=\boldsymbol{\vartheta}_{1}} \\
& \quad \times \mathbf{W}^{-1}\left(\mathbf{s}_{4} \odot \mathbf{n}_{\mathrm{TDOA}-\mathrm{AOA}}\right) \\
& \approx 2\left[\left[\begin{array}{ll}
\mathbf{S}^{T} & \mathbf{H}^{T}
\end{array}\right]+d_{1}^{-1} \boldsymbol{\vartheta}_{1}\left(\left[\begin{array}{ll}
\mathbf{s}_{1}^{T} & \mathbf{0}_{M}^{T}
\end{array}\right]-d_{1}\left[\begin{array}{ll}
\mathbf{1}_{M-1}^{T} & \mathbf{0}_{M}^{T}
\end{array}\right]\right)\right]
\end{aligned}
$$




$$
\begin{aligned}
& \times \mathbf{W}^{-1}\left[\left[\begin{array}{l}
\mathbf{S} \\
\mathbf{H}
\end{array}\right]\left[\begin{array}{l}
1 \\
0
\end{array}\right]+d_{1}^{-1}\left(\left[\begin{array}{c}
\mathbf{s}_{1} \\
\mathbf{0}_{M}
\end{array}\right]-d_{1}\left[\begin{array}{c}
\mathbf{1}_{M-1} \\
\mathbf{0}_{M}
\end{array}\right]\right)\left(x-x_{1}\right)\right] \\
& +2\left[\left[\begin{array}{ll}
\mathbf{S}^{T} & \mathbf{H}^{T}
\end{array}\right]+d_{1}^{-1} \boldsymbol{\vartheta}_{1}\left(\left[\begin{array}{ll}
\mathbf{s}_{1}^{T} & \mathbf{0}_{M}^{T}
\end{array}\right]-d_{1}\left[\begin{array}{ll}
\mathbf{1}_{M-1}^{T} & \mathbf{0}_{M}^{T}
\end{array}\right]\right)\right] \\
& \times d_{1}^{-1} \mathbf{W}^{-1} \mathbf{n}_{\text {TDOA-AOA }}\left(x-x_{1}\right)+2 d_{1}^{-1} \boldsymbol{\vartheta}_{1} \mathbf{n}_{\mathrm{TDOA}}^{T} \mathbf{W}^{-1} \\
& \times\left[\left[\begin{array}{l}
\mathbf{S} \\
\mathbf{H}
\end{array}\right]\left[\begin{array}{l}
1 \\
0
\end{array}\right]+d_{1}^{-1}\left(\left[\begin{array}{c}
\mathbf{s}_{1} \\
\mathbf{0}_{M}
\end{array}\right]-d_{1}\left[\begin{array}{c}
\mathbf{1}_{M-1} \\
\mathbf{0}_{M}
\end{array}\right]\right)\left(x-x_{1}\right)\right] \\
& +\left.2 \frac{\partial}{\partial \breve{x}}\left[\left[\begin{array}{ll}
\mathbf{S}^{T} & \mathbf{H}^{T}
\end{array}\right]+\left(\breve{\boldsymbol{\vartheta}}_{1}^{T} \breve{\vartheta}_{1}\right)^{-1 / 2} \breve{\vartheta}_{1}\left[\begin{array}{ll}
\mathbf{r}_{\mathrm{TDOA}}^{T} & \mathbf{0}_{M}^{T}
\end{array}\right]\right]\right|_{\breve{\vartheta}_{1}=\mathbf{\vartheta}_{1}} \\
& \times \mathbf{W}^{-1}\left(\mathbf{s}_{4} \odot \mathbf{n}_{\text {TDOA-AOA }}\right) \text {. }
\end{aligned}
$$

Taking the expected value on both sides of (B.34) and applying the fact that $E\left[\mathbf{n}_{\mathrm{TDOA}-\mathrm{AOA}}\right]=\mathbf{0}_{2 M-1}$ gives

$$
\begin{aligned}
& \left.E\left[\frac{\partial}{\partial \breve{x}}\left(\frac{\partial J_{\mathrm{TDOA}-\mathrm{AOA}}}{\partial \breve{\boldsymbol{\vartheta}}_{1}}\right)\right]\right|_{\check{\mathfrak{\vartheta}}_{1}=\mathbf{9}_{1}} \\
& \approx 2\left[\left[\begin{array}{ll}
\mathbf{S}^{T} & \mathbf{H}^{T}
\end{array}\right]+d_{1}^{-1} \mathbf{\vartheta}_{1}\left(\left[\begin{array}{ll}
\mathbf{s}_{1}^{T} & \mathbf{0}_{M}^{T}
\end{array}\right]-d_{1}\left[\begin{array}{ll}
\mathbf{1}_{M-1}^{T} & \mathbf{0}_{M}^{T}
\end{array}\right]\right)\right] \\
& \quad \times \mathbf{W}^{-1}\left[\left[\begin{array}{l}
\mathbf{S} \\
\mathbf{H}
\end{array}\right]\left[\begin{array}{l}
1 \\
0
\end{array}\right]+d_{1}^{-1}\left(\left[\begin{array}{c}
\mathbf{s}_{1} \\
\mathbf{0}_{M}
\end{array}\right]-d_{1}\left[\begin{array}{c}
\mathbf{1}_{M-1} \\
\mathbf{0}_{M}
\end{array}\right]\right)\left(x-x_{1}\right)\right] .
\end{aligned}
$$

Similarly, repeating the derivation in (B.33), (B.34), and (B.35) with the variable $\breve{y}$ gives

$$
\begin{aligned}
& \left.E\left[\frac{\partial}{\partial \breve{y}}\left(\frac{\partial J_{\mathrm{TDOA}-\mathrm{AOA}}}{\partial \breve{\boldsymbol{\vartheta}}_{1}}\right)\right]\right|_{\check{\mathfrak{\vartheta}}_{1}=\mathbf{9}_{1}} \\
& \approx 2\left[\left[\begin{array}{ll}
\mathbf{S}^{T} & \mathbf{H}^{T}
\end{array}\right]+d_{1}^{-1} \mathbf{\vartheta}_{1}\left(\left[\begin{array}{ll}
\mathbf{s}_{1}^{T} & \mathbf{0}_{M}^{T}
\end{array}\right]-d_{1}\left[\begin{array}{ll}
\mathbf{1}_{M-1}^{T} & \mathbf{0}_{M}^{T}
\end{array}\right]\right)\right] \\
& \quad \times \mathbf{W}^{-1}\left[\left[\begin{array}{l}
\mathbf{S} \\
\mathbf{H}
\end{array}\right]\left[\begin{array}{l}
0 \\
1
\end{array}\right]+d_{1}^{-1}\left(\left[\begin{array}{c}
\mathbf{s}_{1} \\
\mathbf{0}_{M}
\end{array}\right]-d_{1}\left[\begin{array}{c}
\mathbf{1}_{M-1} \\
\mathbf{0}_{M}
\end{array}\right]\right)\left(y-y_{1}\right)\right] .
\end{aligned}
$$

We also have

$$
\begin{aligned}
& \left.E\left[\frac{\partial^{2} J_{\mathrm{TDOA}-\mathrm{AOA}}}{\partial \breve{\boldsymbol{\vartheta}}_{1} \partial \breve{\boldsymbol{\vartheta}}_{1}^{T}}\right]\right|_{\breve{\vartheta}_{1}=\mathbf{\vartheta}_{1}} \\
& =\left[\left.\left.E\left[\frac{\partial}{\partial \breve{x}}\left(\frac{\partial J_{\mathrm{TDOA}-\mathrm{AOA}}}{\partial \breve{\boldsymbol{\vartheta}}_{1}}\right)\right]\right|_{\breve{\vartheta}_{1}=\boldsymbol{\vartheta}_{1}} E\left[\frac{\partial}{\partial \breve{y}}\left(\frac{\partial J_{\mathrm{TDOA}-\mathrm{AOA}}}{\partial \breve{\boldsymbol{\vartheta}}_{1}}\right)\right]\right|_{\breve{\vartheta}_{1}=\mathbf{\vartheta}_{1}}\right] .
\end{aligned}
$$

Hence substituting (B.35) and (B.36) into (B.37), we get

$$
\begin{aligned}
& \left.E\left[\frac{\partial^{2} J_{\text {TDOA-AOA }}}{\partial \breve{\boldsymbol{\vartheta}}_{1} \partial \breve{\boldsymbol{\vartheta}}_{1}^{T}}\right]\right|_{\check{\mathfrak{\vartheta}}_{1}=\boldsymbol{\vartheta}_{1}} \\
& \approx 2\left[\left[\begin{array}{ll}
\mathbf{S}^{T} & \mathbf{H}^{T}
\end{array}\right]+\boldsymbol{\vartheta}_{1}\left(d_{1}^{-1}\left[\begin{array}{c}
\mathbf{s}_{1} \\
\mathbf{0}_{M}
\end{array}\right]-\left[\begin{array}{c}
\mathbf{1}_{M-1} \\
\mathbf{0}_{M}
\end{array}\right]\right)^{T}\right] \\
& \times \mathbf{W}^{-1}\left[\left[\begin{array}{c}
\mathbf{S} \\
\mathbf{H}
\end{array}\right]+\left(d_{1}^{-1}\left[\begin{array}{c}
\mathbf{s}_{1} \\
\mathbf{0}_{M}
\end{array}\right]-\left[\begin{array}{c}
\mathbf{1}_{M-1} \\
\mathbf{0}_{M}
\end{array}\right]\right) \boldsymbol{\vartheta}_{1}^{T}\right] .
\end{aligned}
$$

Then by substituting (B.32) and (B.38) into (65), the covariance matrix for the MS position estimate $\boldsymbol{\vartheta}_{1}$ is obtained as

$$
\begin{aligned}
\mathbf{C}_{\boldsymbol{\vartheta}_{1}} \approx\{[ & {\left.\left[\begin{array}{ll}
\mathbf{S}^{T} & \mathbf{H}^{T}
\end{array}\right]+\boldsymbol{\vartheta}_{1}\left(d_{1}^{-1}\left[\begin{array}{c}
\mathbf{s}_{1} \\
\mathbf{0}_{M}
\end{array}\right]-\left[\begin{array}{c}
\mathbf{1}_{M-1} \\
\mathbf{0}_{M}
\end{array}\right]\right)^{T}\right] } \\
& \left.\times \mathbf{W}^{-1}\left[\left[\begin{array}{c}
\mathbf{S} \\
\mathbf{H}
\end{array}\right]+\left(d_{1}^{-1}\left[\begin{array}{c}
\mathbf{s}_{1} \\
\mathbf{0}_{M}
\end{array}\right]-\left[\begin{array}{c}
\mathbf{1}_{M-1} \\
\mathbf{0}_{M}
\end{array}\right]\right) \boldsymbol{\vartheta}_{1}^{T}\right]\right\}^{-1} .
\end{aligned}
$$

Substituting $\mathbf{x}-\mathbf{x}_{1}$ back to $\boldsymbol{\vartheta}_{1}$ in (B.32) and (B.39) and applying the fact that $C_{x}=C_{\vartheta_{1}}$ gives (96) and (97).

c.

The Cramér-Rao lower bound (CRLB) gives a lower bound on variance attainable by any unbiased estimators and thus it can serve as a benchmark for the mean square position errors (MSPEs) of the positioning algorithms. To determine it, the key step is to construct the Fisher information matrix (FIM) using the probability density function of the measurements parameterized by the MS position, and the standard procedure for obtaining the CRLB can be found in [21]. When the measurement errors are Gaussian distributed, the FIM for mobile positioning using TDOA measurements, denoted by $\mathbf{I}_{\mathrm{TDOA}}(\mathbf{x})$, is given by $[14,15]$

$$
\mathbf{I}_{\mathrm{TDOA}}(\mathbf{x})=\left.\left[\frac{\partial \mathbf{f}_{\mathrm{TDOA}}}{\partial \breve{\mathbf{x}}}\right]^{T} \mathbf{C}_{\mathbf{n}, \mathrm{TDOA}}^{-1}\left[\frac{\partial \mathbf{f}_{\mathrm{TDOA}}}{\partial \breve{\mathbf{x}}}\right]\right|_{\breve{\mathbf{x}}=\mathbf{x}},
$$

where 


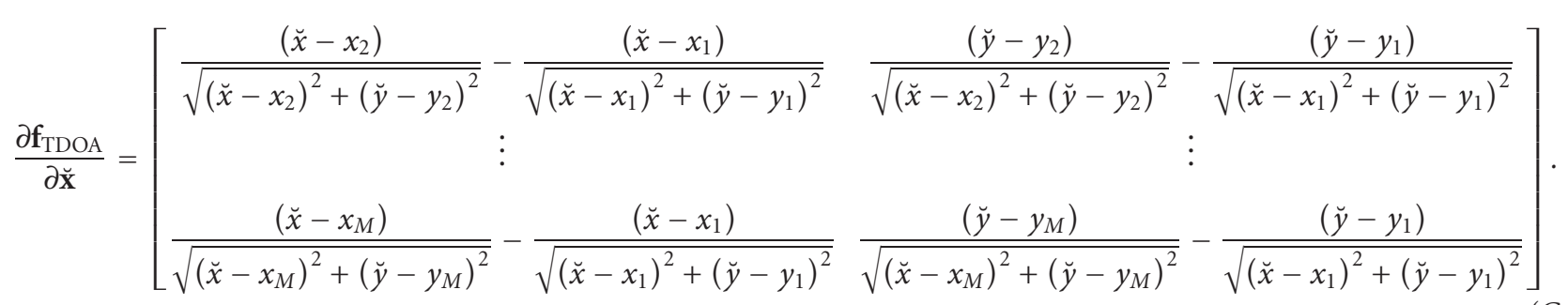

Similarly, the FIMs for RSS, AOA, and TDOA-AOA hybrid based mobile positioning, denoted by $\mathbf{I}_{\mathrm{RSS}}(\mathbf{x}), \mathbf{I}_{\mathrm{AOA}}(\mathbf{x})$, and $\mathbf{I}_{\mathrm{TDOA}-\mathrm{AOA}}(\mathbf{x})$, respectively, are given by

$$
\begin{aligned}
\mathbf{I}_{\mathrm{RSS}}(\mathbf{x})= & {\left[\frac{\partial \mathbf{f}_{\mathrm{RSS}}}{\partial \breve{\mathbf{x}}}\right]^{T} } \\
& \times\left.\mathbf{C}_{\mathbf{n}, \mathrm{RSS}}^{-1}\left[\frac{\partial \mathbf{f}_{\mathrm{RSS}}}{\partial \breve{\mathbf{x}}}\right]\right|_{\check{\mathbf{x}}=\mathbf{x}},
\end{aligned}
$$

$$
\mathbf{I}_{\mathrm{AOA}}(\mathbf{x})=\left.\left[\frac{\partial \mathbf{f}_{\mathrm{AOA}}}{\partial \breve{\mathbf{x}}}\right]^{T} \mathbf{C}_{\mathbf{n}, \mathrm{AOA}}^{-1}\left[\frac{\partial \mathbf{f}_{\mathrm{AOA}}}{\partial \breve{\mathbf{x}}}\right]\right|_{\breve{\mathbf{x}}=\mathbf{x}},
$$

$\mathbf{I}_{\mathrm{TDOA}-\mathrm{AOA}}(\mathbf{x})=\left.\left[\frac{\partial \mathbf{f}_{\mathrm{TDOA}-\mathrm{AOA}}}{\partial \breve{\mathbf{x}}}\right]^{T} \mathbf{C}_{\mathbf{n}, \mathrm{TDOA}-\mathrm{AOA}}^{-1}\left[\frac{\partial \mathbf{f}_{\mathrm{TDOA}-\mathrm{AOA}}}{\partial \breve{\mathbf{x}}}\right]\right|_{\check{\mathbf{x}}=\mathbf{x}}$,

where

$$
\begin{aligned}
& \frac{\partial \mathbf{f}_{\mathrm{RSS}}}{\partial \breve{\mathbf{x}}}=\left[\begin{array}{cc}
a\left(\breve{x}-x_{1}\right)\left[\left(\breve{x}-x_{1}\right)^{2}+\left(\breve{y}-y_{1}\right)^{2}\right]^{a / 2-1} & a\left(\breve{y}-y_{1}\right)\left[\left(\breve{x}-x_{1}\right)^{2}+\left(\breve{y}-y_{1}\right)^{2}\right]^{a / 2-1} \\
\vdots & \vdots \\
a\left(\breve{x}-x_{M}\right)\left[\left(\breve{x}-x_{M}\right)^{2}+\left(\breve{y}-y_{M}\right)^{2}\right]^{a / 2-1} & a\left(\breve{y}-y_{M}\right)\left[\left(\breve{x}-x_{M}\right)^{2}+\left(\breve{y}-y_{M}\right)^{2}\right]^{a / 2-1}
\end{array}\right], \\
& \frac{\partial \mathbf{f}_{\mathrm{AOA}}}{\partial \breve{\mathbf{x}}}=\left[\begin{array}{cc}
-\frac{\left(\breve{y}-y_{1}\right)}{\left(\breve{x}-x_{1}\right)^{2}+\left(\breve{y}-y_{1}\right)^{2}} & \frac{\left(\breve{x}-x_{1}\right)}{\left(\breve{x}-x_{1}\right)^{2}+\left(\breve{y}-y_{1}\right)^{2}} \\
\vdots & \vdots \\
-\frac{\left(\breve{y}-y_{M}\right)}{\left(\breve{x}-x_{M}\right)^{2}+\left(\breve{y}-y_{M}\right)^{2}} & \frac{\left(\breve{x}-x_{M}\right)}{\left(\breve{x}-x_{M}\right)^{2}+\left(\breve{y}-y_{M}\right)^{2}}
\end{array}\right] \\
& \frac{\partial \mathbf{f}_{\mathrm{TDOA}-\mathrm{AOA}}}{\partial \breve{\mathbf{x}}}=\left[\begin{array}{c}
\frac{\partial \mathbf{f}_{\mathrm{TDOA}}}{\partial \breve{\mathbf{x}}} \\
\frac{\partial \mathbf{f}_{\mathrm{AOA}}}{\partial \breve{\mathbf{x}}}
\end{array}\right] .
\end{aligned}
$$

It is noted that $\mathbf{I}_{\mathrm{TOA}}(\mathbf{x})$ can be computed from $\mathbf{I}_{\mathrm{RSS}}(\mathbf{x})$ in (C.3) by putting $a=1$. Then the CRLBs, namely, $\mathrm{CRLB}_{\mathrm{TDOA}}(\mathbf{x}), \mathrm{CRLB}_{\mathrm{RSS}}(\mathbf{x}), \mathrm{CRLB}_{\mathrm{AOA}}(\mathbf{x}), \mathrm{CRLB}_{\text {TDOA-AOA }}(\mathbf{x})$, and $\mathrm{CRLB}_{\mathrm{TOA}}(\mathbf{x})$ are obtained from the diagonal elements of the inverses of the corresponding FIMs.

\section{ACKNOWLEDGMENTS}

The authors thank Mr. K. W. Chan for his help in developing the nonlinear least squares approach. This work was supported by a grant from the Research Grants Council of the Hong Kong Special Administrative Region, China (Project No. CityU 1119/01E).

\section{REFERENCES}

[1] CC Docket no. 94-102, "Revision of the Commissions Rules to Ensure Compatibility with Enhanced 911 Emergency Calling Systems, RM-8143," July, 1996.

[2] C. Drane, M. Macnaughtan, and C. Scott, "Positioning GSM telephones," IEEE Communications Magazine, vol. 36, no. 4, pp. 46-54, 59, 1998.

[3] H. Koshima and J. Hoshen, "Personal locator services emerge," IEEE Spectrum, vol. 37, no. 2, pp. 41-48, 2000.

[4] Y. Zhao, "Mobile phone location determination and its impact on intelligent transportation systems," IEEE Transactions on Intelligent Transportation Systems, vol. 1, no. 1, pp. 55-64, 2000. 
[5] D. Porcino, "Performance of a OTDOA-IPDL positioning receiver for 3GPP-FDD mode," in Proceedings of the IEE 2 nd International Conference on $3 G$ Mobile Communication Technologies (3G'01), pp. 221-225, London, UK, March 2001.

[6] J. J. Caffery Jr., Wireless Location in CDMA Cellular Radio Systems, Kluwer Academic, Boston, Mass, USA, 2000.

[7] J. C. Liberti and T. S. Rappaport, Smart Antennas for Wireless Communications: IS-95 and Third Generation CDMA Applications, Prentice-Hall, Upper Saddle River, NJ, USA, 1999.

[8] M. McGuire and K. N. Plataniotis, "A comparison of radiolocation for mobile terminals by distance measurements," in Proceedings of International Conference on Wireless Communications, pp. 1356-1359, 2000.

[9] J. J. Caffery Jr. and G. L. Stuber, "Subscriber location in CDMA cellular networks," IEEE Transactions on Vehicular Technology, vol. 47, no. 2, pp. 406-416, 1998.

[10] M. A. Spirito, "On the accuracy of cellular mobile station location estimation," IEEE Transactions on Vehicular Technology, vol. 50, no. 3, pp. 674-685, 2001.

[11] W. H. Foy, "Position-location solutions by Taylor-series estimation," IEEE Transactions on Aerospace and Electronic Systems, vol. 12, no. 2, pp. 187-194, 1976.

[12] D. J. Torrieri, "Statistical theory of passive location systems," IEEE Transactions on Aerospace and Electronic Systems, vol. 20, pp. 183-197, 1984.

[13] J. O. Smith and J. S. Abel, "Closed-form least-squares source location estimation from range-difference measurements," IEEE Transactions on Acoustics, Speech, and Signal Processing, vol. 35, no. 12, pp. 1661-1669, 1987.

[14] Y. T. Chan and K. C. Ho, "A simple and efficient estimator for hyperbolic location," IEEE Transactions on Signal Processing, vol. 42, no. 8, pp. 1905-1915, 1994.

[15] Y. Huang, J. Benesty, G. W. Elko, and R. M. Mersereati, "Realtime passive source localization: a practical linear-correction least-squares approach," IEEE Transactions on Speech and Audio Processing, vol. 9, no. 8, pp. 943-956, 2001.

[16] A. Pages-Zamora, J. Vidal, and D. R. Brooks, "Closed-form solution for positioning based on angle of arrival measurements," in Proceedings of the 13th IEEE International Symposium on Personal, Indoor and Mobile Radio Communications (PIMRC '02), vol. 4, pp. 1522-1526, Lisbon, Portugal, September 2002.

[17] L. Cong and W. Zhuang, "Hybrid TDOA/AOA mobile user location for wideband CDMA cellular systems," IEEE Transactions on Wireless Communications, vol. 1, no. 3, pp. 439-447, 2002.

[18] H. C. So and S. P. Hui, "Constrained location algorithm using TDOA measurements," IEICE Transactions on Fundamentals of Electronics, Communications and Computer Sciences, vol. E86A, no. 12, pp. 3291-3293, 2003.

[19] K. W. Cheung, H. C. So, W.-K. Ma, and Y. T. Chan, "Received signal strength based mobile positioning via constrained weighted least squares," in Proceedings of the IEEE International Conference on Acoustic, Speech and Signal Processing (ICASSP '03), vol. 5, pp. 137-140, Hong Kong, April 2003.

[20] K. W. Cheung, H. C. So, W.-K. Ma, and Y. T. Chan, "Least squares algorithms for time-of-arrival-based mobile location," IEEE Transactions on Signal Processing, vol. 52, no. 4, pp. 1121 1130, 2004.

[21] S. M. Kay, Fundamentals of Statistical Signal Processing: Estimation Theory, Prentice-Hall, Englewood Cliffs, NJ, USA, 1993.

[22] H.-L. Song, "Automatic vehicle location in cellular communications systems," IEEE Transactions on Vehicular Technology, vol. 43, no. 4, pp. 902-908, 1994.
[23] J. Vidal, M. Najar, and R. Jativa, "High resolution time-ofarrival detection for wireless positioning systems," in Proceedings of 56th IEEE Vehicular Technology Conference (VTC '02), vol. 4, pp. 2283-2287, Vancouver, BC, Canada, September 2002.

[24] J. Riba and A. Urruela, "A robust multipath mitigation technique for time-of-arrival estimation," in Proceedings of 56th IEEE Vehicular Technology Conference (VTC '02), vol. 4, pp. 2263-2267, Vancouver, BC, Canada, September 2002.

[25] S. Al-Jazzar, J. J. Caffery Jr., and H.-R. You, "A scattering model based approach to NLOS mitigation in TOA location systems," in Proceedings of 55th IEEE Vehicular Technology Conference (VTC '02), vol. 2, pp. 861-865, Birmingham, Ala, USA, May 2002.

[26] M. P. Wylie-Green and S. S. Wang, "Robust range estimation in the presence of the non-line-of-sight error," in Proceedings of 54th IEEE Vehicular Technology Conference (VTC '01), vol. 1, pp. 101-105, Atlantic City, NJ, USA, September 2001.

[27] N. Patwari, A. O. Hero III, M. Perkins, N. S. Correal, and R. J. O'Dea, "Relative location estimation in wireless sensor networks," IEEE Transactions on Signal Processing, vol. 51, no. 8, pp. 2137-2148, 2003.

[28] K. W. Cheung and H. C. So, "A multidimensional scaling framework for mobile location using time-of-arrival measurements," IEEE Transactions on Signal Processing, vol. 53, no. 2, pp. 460-470, 2005.

[29] T. K. Moon and W. C. Stirling, Mathematical Methods and Algorithms for Signal Processing, Prentice-Hall, Upper Saddle River, NJ, USA, 2000.

K. W. Cheung was born in Hong Kong. He received the B.Eng. degree with first class honors in electrical and electronic engineering from Imperial College of Science, Technology \& Medicine, University of London, in 2001 and the M.Phil. degree in computer engineering and information technology from the City University of Hong Kong in 2004. From October to November 2001, he was a Research Assistant in the Department of Computer Engineering \& Information Technology at the City University of Hong Kong. He is currently working in Hong Kong Science \& Technology Parks. His research interests are in array signal processing, and developing efficient methods in radiolocation for mobile terminals. Mr. Cheung is an Associate Member of Institution of Electrical Engineers in UK and the Hong Kong Institution of Engineers.

H. C. So was born in Hong Kong. He obtained the B.Eng. degree from City University of Hong Kong and the Ph.D. degree from The Chinese University of Hong Kong, both in electronic engineering, in 1990 and 1995, respectively. From 1990 to 1991, he was an electronic engineer at the Research \& Development Division of Everex Systems Engineering Ltd., Hong Kong. During 1995-1996, he worked as a postdoctoral

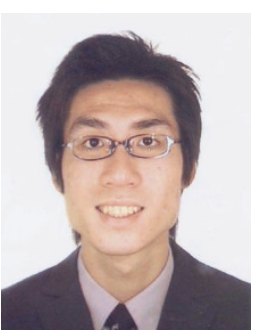
fellow at The Chinese University of Hong Kong. From 1996 to 1999, he was a Research Assistant Professor at the Department of Electronic Engineering, City University of Hong Kong. Currently he is an Associate Professor in the Department of Electronic Engineering at City University of Hong Kong. His research interests include 
adaptive filter theory, detection and estimation, wavelet transform, and signal processing for communications and multimedia.

W.-K. Ma obtained the B.Eng. (with first class honors) in electrical and electronic engineering from the University of Portsmouth, Portsmouth, UK, in 1995. He received the M.Phil. and Ph.D. degrees, both in electronic engineering, from The Chinese University of Hong Kong (CUHK), Hong Kong, in 1997 and 2001, respectively. Since August 2005, he has been an Assistant Professor in the Department of Electrical

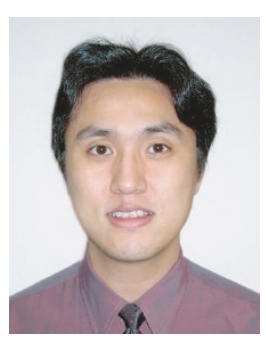
Engineering and the Institute of Communications Engineering, the National Tsing Hua University, Taiwan. Previously he has held research positions at McMaster University, Canada, CUHK, Hong Kong, and the University of Melbourne, Australia. His research interests are in signal processing for communications and statistical signal processing. Dr. Ma's Ph.D. dissertation was commended to be "of very high quality and well-deserved honorary mentioning" by the Faculty of Engineering, CUHK, in 2001.

Y. T. Chan was born in Hong Kong, and received his electrical engineering education in Canada. His Bachelor's and Master's degrees are from Queen's University, and his $\mathrm{Ph} . \mathrm{D}$. degree from the University of New Brunswick. He was an engineer with Nortel Networks and has been a Professor in the Electrical and Computer Engineering Department at the Royal Military College of

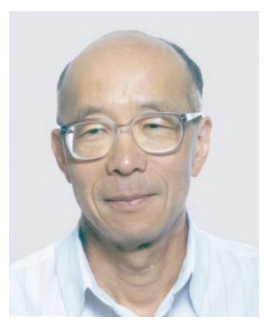
Canada, serving as Head of the department from 1994 to 2000. From 2002 to 2005, he was a Visiting Professor at the Electronic Engineering Department of The Chinese University of Hong Kong. Presently he is an Adjunct Professor at the Royal Military College. His research interests are in detection, estimation, localization, and tracking. Kluwer Academic Publishers published his text Wavelet Basics in 1994. Dr. Chan was an Associate Editor of the IEEE Transactions on Signal Processing, the Technical Chair of ICASSP-84, General Chair of ICASSP-91, Vice Chair of ICASSP-03, and Social Chair of ICASSP-04. He directed a NATO ASI in 1988. 